\title{
Heavy quark effective theory beyond perturbation theory: renormalons, the pole mass and the residual mass term
}

\author{
M. Beneke ${ }^{\mathrm{a}}$, V.M. Braun ${ }^{\mathrm{b}, 1}$ \\ ${ }^{a}$ Randall Laboratory of Physics, University of Michigan, Ann Arbor, MI 48109, USA \\ ${ }^{\mathrm{b}}$ Max-Planck-Institut für Physik, -Werner-Heisenberg-Institut-, D-80805 Munich, Germany
}

Received 16 March 1994; accepted 28 April 1994

\begin{abstract}
We study the asymptotic behaviour of the perturbative series in the heavy quark effective theory (HQET) using the $1 / N_{f}$ expansion. We find that this theory suffers from an ultraviolet renormalon problem, corresponding to a non-Borel-summable behaviour of perturbation series in large orders, and leading to a principal nonperturbative ambiguity in its definition. This ambiguity is related to an infrared renormalon in the pole mass and can be understood as the necessity to include the residual mass term $\delta m$ in the definition of HQET, which must be considered as ambiguous (and possibly complex), and is required to cancel the ultraviolet renormalon singularity generated by the perturbative expansion. The formal status of $\delta m$ is thus identical to that of condensates in the conventional short-distance expansion of correlation functions in QCD. The status of the pole mass of a heavy quark, the operator product expansion for inclusive decays, and QCD sum rules in the HQET are discussed in this context.
\end{abstract}

\section{Introduction}

The past few years have witnessed considerable progress in understanding the decays of hadrons containing a single heavy quark in the kinematical regime, where the heavy quark is almost on-shell. This progress has mainly been achieved through the implementation of an effective field theory, which eliminates the heavy quark as a dynamical degree of freedom [1-3]. In the infinite mass limit, the effective theory unravels new

\footnotetext{
${ }^{1}$ On leave of absence from St. Petersburg Nuclear Physics Institute, 188350 Gatchina, Russia.
} 
symmetries of QCD [4], while providing a systematic approach to treat corrections to this limit, which are suppressed by inverse powers of the heavy quark mass $m_{Q}$. The number of independent form factors governing the decays of heavy hadrons is greatly reduced by these symmetries, which allows us to relate the width and spectra of various decays. A peculiar property of heavy quark effective theory (HQET) is that each effective quark field is labelled by the velocity four-vector $v_{\mu}$ of the heavy quark, which is conserved by the strong interactions in the limit of an infinitely heavy quark. Deep connections have been pointed out [5] between HQET and the dynamics of Wilson loops [6-11], related to the infrared behaviour of perturbative QCD and the factorization of soft divergences.

Given the importance of HQET for different branches of phenomenology, it is instructive to investigate its status as a quantum field theory. Thus the leading-order effective Lagrangian,

$$
\mathcal{L}_{\text {eff }}=\bar{h}_{v} i v \cdot D h_{v}+\mathcal{L}_{\text {light }},
$$

where $v$ labels the velocity of the heavy quark and $\mathcal{L}_{\text {light }}$ denotes the Lagrangian for the light degrees of freedom, has been proven to be renormalizable to all orders in perturbation theory [12] (see also Refs. [7-11]). The main objective of this paper is to investigate the nonperturbative properties of the theory defined by the Lagrangian in Eq. (1.1), which show up in the asymptotic behaviour of perturbation theory. To this purpose we study the structure of singularities of correlation functions in HQET in the complex plane of the Borel transform with respect to the strong coupling, an approach that has been pioneered in Ref. [13] in its application to QCD. Our main finding is that apart from the usual sequences of infrared (IR) and ultraviolet (UV) renormalons, which one expects to be inherited from QCD, the HQET suffers from an additional series of UV renormalons, which are non-Borel-summable. Thus the Lagrangian $\mathcal{L}_{\text {eff }}$ as it stands does not define a respectable theory, since it is plagued by an ultraviolet renormalon problem in the same way as, e.g., QED [14], albeit for different reasons ${ }^{2}$. In other words, the high momentum region of internal integrations in Feynman diagrams renders the perturbative expansion of Green functions so badly divergent in large orders as to obstruct their unambiguous definition beyond perturbation theory.

At this point it is helpful to keep in mind that despite the sophisticated terminology prevailing the field of large-order perturbation theory, the physics reflected in the emergence of renormalons is usually simple and can be understood without recourse to the asymptotics of perturbation theory. In QED, for instance, the UV renormalons appear as a disguise of the good old Landau ghost. Whereas thus the UV renormalons are presumably fatal for QED as a viable theory (unless it becomes embedded in a larger nonabelian group), this is of course not so for the heavy quark limit of QCD, since the leading effective Lagrangian, Eq. (1.1), has to be supplemented by a tower of nonrenormalizable interactions, suppressed by inverse powers of the heavy quark mass, as well as renormalization of their coefficients taking into account the QCD interactions on scales above $m_{Q}$. It is precisely this separation of effects on different distance scales

\footnotetext{
${ }^{2}$ Ultraviolet renormalons appear in QCD as well, but since they are Borel-summable in this case, they are usually not considered as a "problem".
} 
into coefficient functions and matrix elements that introduces infrared renormalons into the coefficient functions and ultraviolet renormalons into the matrix elements of composite operators at the same position in the Borel plane, since the virtual particles inside the loops do not respect the constraint $k>m_{Q}, k<m_{Q}$, respectively, on their internal momentum. This phenomenon is well-known from the short-distance expansion of correlation functions in QCD or the $\mathrm{O}(N)$-nonlinear sigma model [15], though to our knowledge it has never been made explicit in any calculation. However, this observation alone is not sufficient to cure the UV renormalon disease in the HQET. The crucial point is that the leading order effective Lagrangian, Eq. (1.1), extracts the correct dependence on the heavy quark mass of the Green functions in full QCD only after subtraction of a term that scales with $m_{Q}$ (consider, to be definite, the inverse heavy quark propagator as in Ref. [2]). This implies a choice of $m_{Q}$ that coincides with the pole mass of the heavy quark to all orders in perturbation theory, but is arbitrary otherwise. Here the subtleties arise.

In a confining theory like QCD the $S$-matrix elements have no poles corresponding to a physical quark and therefore there is no natural choice of the expansion parameter $m_{Q}$. Indeed, the mass of the lightest meson containing the heavy quark flavour would serve this purpose as well as any other parameter that differs from the meson mass by an amount exponentially small in the coupling. This obvious ambiguity has been known from the very beginnings of HQET and prompted the authors of Ref. [16] to introduce the concept of a residual mass term, $-\delta m \bar{h}_{v} h_{v}$, to be added to the Lagrangian of Eq. (1.1). The residual mass term, being of order $A$, where $\Lambda$ is a characteristic low energy scale of QCD, will enter the expressions of HQET, once one leaves the framework of perturbation theory. The predictions of HQET must be independent of the choice of $m_{Q}$. Indeed, it has been shown [16] that the residual mass term enters the matrix elements of HQET through the combination $\bar{\Lambda}-\delta m$ only, where $m_{P}-m_{Q}=\bar{\Lambda}+O\left(1 / m_{Q}\right)$ is the difference between the mass $m_{P}$ of the meson under consideration and the heavy quark expansion parameter in the infinite mass limit. This combination is clearly invariant under the choice of $m_{Q}$, thus apparently justifying the choice $\delta m=0$ implicit in most works on HQET. This conclusion is incorrect. As will be seen in the subsequent sections, the pole mass develops an IR renormalon, which, when subtracted in the construction of HQET, necessitates the inclusion of a residual mass term as a "remnant" of this IR renormalon. If we insist on a formal expansion in $\alpha\left(m_{Q}\right)$ and $\Lambda / m_{Q}$, the residual mass term must be considered as ambiguous (and possibly complex) and this ambiguity is required to cancel the UV renormalon in the matrix elements of operators in the HQET. To express this statement in a different way: though $\bar{\Lambda}-\delta m$ is invariant under the choice of $m_{Q}$, it is not invariant under the choice of a summation prescription for the divergent perturbative expansions in the HQET. We wish to note here that the inherent ambiguity of the quantity $\bar{A}-\delta m$ has been conceptually realized in Ref. [17], where some of our results are anticipated. The formal status of $\bar{A}-\delta m$ is thus identical to that of condensates in the conventional short-distance expansion of correlation functions in QCD.

This analogy may be pursued further. It has been known for a long time that the computation of dimensionful parameters like condensates is a very difficult task for lattice gauge theories, due to mixing with lower dimensional operators, which manifests 
itself in power divergences in the lattice spacing, as the latter is taken to zero $[18,19]$. The power divergences require a nonperturbative regularization procedure, which is essentially equivalent to fixing a specific summation prescription for the perturbative expansion. This connects power divergences in lattice gauge theory to renormalons in the continuum theory, where we might note in addition that mixing between operators of different dimension occurs in the continuum precisely through the appearance of renormalons. Our observation that $\bar{A}-\delta m$ (and similar parameters in higher orders of the $1 / m_{Q}$ expansion) is ambiguous is therefore completely consistent with the observation of power divergences in the lattice version of HQET that have been reported in Ref. [20], and which turn out to be an obstacle to the determination of HQET matrix elements on the lattice starting at order $1 / m_{Q}$.

The study of the asymptotics of the perturbative series in HQET is a rather formal subject, but allows us to draw several immediate conclusions of practical importance. The first of them concerns inclusive $B$-decays, which are receiving much attention presently. It has been shown $[21,22]$ that nonperturbative corrections to total inclusive widths can be studied using the operator product expansion technique, and are suppressed by two powers of the large $b$-quark mass. It is widely believed that $O\left(1 / m_{b}\right)$ corrections to the total widths are absent, provided the latter are expressed in terms of the pole $b$-quark mass, see Ref. [23] for the clearest presentation of this conviction. The nonperturbative $1 / m_{b}^{2}$ corrections turn out to be very small in reality, and this result has already triggered attempts to determine the $b$-quark pole mass from the data on the total decay rates [24]. Our results show that the absence of $1 / m_{b}$ corrections is illusory. Different prescriptions for the summation of the perturbative series defining the pole mass introduce a principle uncertainty in the predictions for the decay rates. The data on the total inclusive widths can not be used to determine the pole mass, but rather to define it. This definition is not worse, but also not better than any other phenomenological definition, e.g. from the QCD sum rules for the $B$-mesons or $T$ family, see Refs. [25-27]. The existing estimates for the value of the $b$-quark pole mass fall in the range $m_{b}=4.55-4.85 \mathrm{GeV}$, indicating a possible uncertainty of the order of a few hundred $\mathrm{MeV}$. We find very similar values for the intrinsic uncertainty in the pole mass from our results on the ambiguity in the summation of the perturbative series.

Second, we address the QCD sum rule technique [28], which has been used to obtain quantitative estimates for $\bar{A}$ and other observables in HQET (see Ref. [29] for a review). Our analysis suggests that the residual mass term should be included as an additional phenomenological parameter (like the condensates) into the expansion of correlation functions in the HQET. The effect of this parameter is, however, very specific. We show that, loosely speaking, the renormalons associated with the residual mass term can be "summed up" and result in an ambiguity in the momentum scale of the correlation function, so that quantities like the $B$-meson decay constant $f_{B}$ and the heavy quark kinetic energy [30] are formally not affected.

The most important question is whether this ambiguity is important numerically. Again, we might appeal to the more familiar situation of the short-distance expansion of correlation functions in QCD, where the gluon condensate, e.g., has been determined despite its ambiguity, observing that its actual value is "large" in the sense that the IR renormalon of perturbation theory may be ignored. It is however very important to recall 
that this can be justified only a posteriori by the very success of the sum rules. There is no guarantee that the same conclusion applies to the parameters of the heavy quark expansion.

The further presentation is organized as follows. In Sect. 2 we find it useful to recapitulate some facts on the operator product expansion and IR renormalons of the correlation function of light quark currents. This will also allow us to introduce the basic notions in dealing with large-order perturbation theory that will be needed later on. In Sect. 3 we study in detail the perturbative expansion of the inverse propagator of a massive quark in QCD and its matching onto the heavy quark expansion. New IR and UV renormalons will be seen to emerge in this limit. Calculations to all orders in perturbation theory are performed in an expansion in $1 / N_{f}$, with $N_{f}$ the number of light flavours. We move to the consideration of the correlation function of heavy-light currents in Sect. 4, where the analytic properties of its Borel transform are obtained and discussed. Sect. 5 is devoted to applications of our results to the practice of QCD sum rule calculations and in Sect. 6 we present a summary and conclusions.

Two appendices deal with some more technical issues. In Appendix A we show, how the renormalization of the Borel transform proceeds in the leading order of the $1 / N_{f}$ expansion. For simplicity of notations, the explicit derivation is given for the selfenergy of the heavy quark. In Appendix B we compute the scalar two-loop integral with arbitrary power of the propagator of the interchanged scalar. The singularity structure of this integral is required to obtain the analytic structure of the Borel-transformed correlation function discussed in Sect. 4.

\section{IR renormalons and the short-distance expansion of light quark current correlation functions}

The best studied (see e.g. Refs. [31,32] for two recent expositions) and most transparent quantity to exhibit the relation of the IR asymptotics of perturbation series to the short-distance expansion is provided by the correlation function

$$
\begin{aligned}
\Pi_{\mu \nu}(q) & =\left(q_{\mu} q_{\nu}-q^{2} g_{\mu \nu}\right) \Pi\left(Q^{2}\right) \\
& =i \int \mathrm{d}^{4} x e^{i q x}\left\langle 0\left|T\left\{j_{\mu}^{\dagger}(x) j_{\nu}(0)\right\}\right| 0\right\rangle, \quad Q^{2}=-q^{2}
\end{aligned}
$$

of two vector currents $j_{\mu}(x)=\bar{q}(x) \gamma_{\mu} q(x)$ of light, i.e. massless, quarks. It is useful to recall this relation in detail, since the same concepts will recur in the more intricate context of the heavy quark expansion. We hope that the yet inevitable sketchiness of our presentation does not appall the more rigorous minded readers.

Let us first focus on the perturbative expansion of $\Pi\left(Q^{2} / \mu^{2}, \alpha(\mu)\right)$ in the strong coupling. One may easily identify one source of divergence of this expansion in large orders. To this end, one investigates the diagram of Fig. 1 with the gluon line dressed by a chain of fermion, gluon and ghost loops, summation of which is essentially equivalent to placing the running coupling $g(k)$ at the vertices, where $k$ is the momentum which 


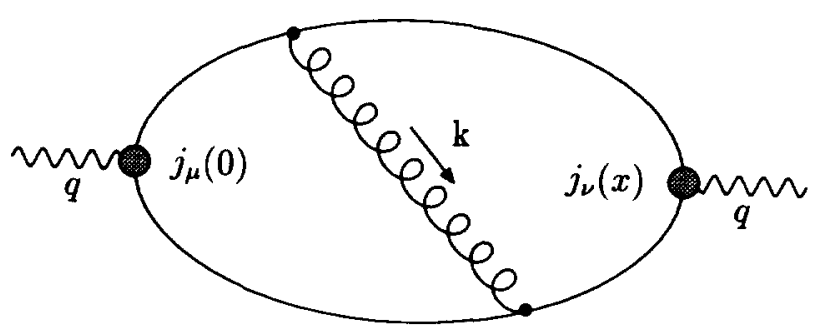

Fig. 1. Two-loop diagram for the correlation function of vector currents.

flows through the gluon line ${ }^{3}$. Since we are interested in the contribution from soft gluons, $k \ll Q$, after performing all other integrations, we expand in $k^{2} / Q^{2}$ and obtain

$$
\begin{aligned}
\Pi\left(\frac{Q^{2}}{\mu^{2}}, \alpha(\mu)\right) & \ni \int^{\lambda} \frac{\mathrm{d} k^{2}}{k^{2}}\left(\frac{k^{2}}{Q^{2}}\right)^{m} \alpha(k) \\
& =\sum_{n} \alpha(Q)^{n+1} \int^{\lambda} \frac{\mathrm{d} k^{2}}{k^{2}}\left(\frac{k^{2}}{Q^{2}}\right)^{m}\left(\beta_{0} \ln \frac{k^{2}}{Q^{2}}\right)^{n},
\end{aligned}
$$

where $m$ is a natural number, " $\ni$ " denotes a contribution to the asymptotic behaviour, which need not be the dominant one, and $\lambda$ regularizes the UV divergence introduced through the expansion in $k^{2} / Q^{2}$. The first coefficient of the $\beta$-function, $\beta_{0}$, is negative in our definition. The logarithmic behaviour of the running coupling drives the gluon line to increasingly softer momentum, $k \sim Q \mathrm{e}^{-n /(2 m)}$, as $n$ becomes large. At the same time, the logarithm is large in this regime, no matter what (fixed!) renormalization scale one chooses (we have taken $\mu=Q$ for convenience). As a result, a factorial divergence of the perturbative expansion

$$
\Pi\left(\frac{Q^{2}}{\mu^{2}}, \alpha(\mu)\right) \equiv \sum_{n} \Pi_{n}\left(\frac{Q^{2}}{\mu^{2}}\right) \alpha(\mu)^{n+1} \ni \sum_{n}\left(-\frac{\beta_{0}}{m}\right)^{n} n ! \alpha(Q)^{n+1}
$$

arises $^{4}$. We may still make progress and define the Borel transform of $\Pi$ by

$$
\tilde{\Pi}\left(\frac{Q^{2}}{\mu^{2}}, t\right) \equiv \sum_{n} \Pi_{n}\left(\frac{Q^{2}}{\mu^{2}}\right) \frac{t^{n}}{n !} .
$$

In favourable circumstances [33], $\Pi$ can be recovered despite its divergent expansion from the integral representation

\footnotetext{
${ }^{3}$ This statement is strictly true only in QED. In QCD, dressing of a gluon by a chain of bubbles is not a gauge-invariant procedure and one must leave the framework of bubble summation to obtain the correct coefficient $\beta_{0}$ in front of the logarithm in Eq. (2.2).

${ }^{4}$ For completeness, we note that a similar, but sign-alternating divergence proportional to $\left(\beta_{0} / m\right)^{n} n$ ! is present by the same reasoning, applied to the ultraviolet region, $k \gg Q$.
} 


$$
\Pi\left(\frac{Q^{2}}{\mu^{2}}, \alpha(\mu)\right)=\int_{0}^{\infty} \mathrm{d} t \mathrm{e}^{-t / \alpha(\mu)} \tilde{\Pi}\left(\frac{Q^{2}}{\mu^{2}}, t\right) .
$$

However, from Eq. (2.3) one infers that the Borel transform $\tilde{\Pi}$ has (IR renormalon) singularities at $t=-m / \beta_{0}$ on the integration contour. The naive Borel summation fails in QCD and does not define $\Pi$ unambiguously. As a measure of this ambiguity one may take the difference between the contour prescriptions above and under the real axis in the complex Borel plane. One then concludes that within perturbation theory we can account for the infrared domain only up to terms of order

$$
\delta \Pi_{\mathrm{IR}}\left(Q^{2}\right) \ni \exp \left(\frac{m}{\beta_{0} \alpha(Q)}\right) \sim\left(\frac{\Lambda^{2}}{Q^{2}}\right)^{m} .
$$

This deficiency of perturbation theory has a profound reason. In the real world, quarks and gluons are confined into hadrons and one does not expect this phenomenon to occur as a mere consequence of summation of the perturbative series and analytic continuation to the physical region. If QCD is to have any significance for the real world, perturbation theory must be incomplete and the IR renormalons are just a reminder that nonperturbative terms must be added. Moreover, the location of singularities on the positive real Borel axis traces the order of magnitude of these effects.

In case of correlation functions at deep euclidean momentum, the framework for a systematic incorporation of nonperturbative effects is the short-distance expansion (SDE) of the operator product $j_{\mu}(x) j_{\nu}(0)$, which leads to the improved expression [28]

$$
\begin{aligned}
\Pi\left(\frac{Q^{2}}{\mu^{2}}, \alpha(\mu)\right)= & \sum_{n} p_{n}\left(\frac{Q^{2}}{\mu^{2}}\right) \alpha(\mu)^{n+1}+C_{G G}\left(\frac{Q^{2}}{\mu^{2}}, \alpha(\mu)\right) \frac{1}{Q^{4}}\left\langle 0\left|\frac{\alpha}{\pi} G G\right| 0\right\rangle(\mu) \\
& +O\left(\frac{1}{Q^{6}}\right)
\end{aligned}
$$

where the new input is given by the gluon condensate and its Wilson coefficient function and higher power-suppressed terms involve the vacuum expectation value of higher dimensional operators. This representation is supposed to yield an unambiguous answer for $\Pi$, including all nonperturbative effects up to terms of order $1 / Q^{6}$. How is this possible in view of the above ambiguities inherent to the perturbative contribution due to IR renormalons? This question can be answered from a formal and a practical position and we choose to begin with the first.

The nonperturbative definition of condensates is indeed a delicate problem. We may pick a physical cutoff, in which case the operator $\alpha / \pi G G$ can mix with lower dimensional ones, the unit operator in particular. In order to define the normal product, one must subtract these contributions, which is an ambiguous procedure due to the occurrence of renormalons in their series in the limit, when the cutoff is removed. To fix an exponentially small in $\alpha$ term like $\langle 0|\alpha / \pi G G| 0\rangle$, one must first give a meaning to the divergent perturbative expansions in lower dimensional operators, which may be complex, depending on the contour prescription for the singularities in their Borel representation. 
Without this specification, we are thus led to the notion of ambiguous (and possibly complex) condensates ${ }^{5}$, although by no means this implies that the correlation function $I I$ is ambiguous (or complex). To the contrary, this imaginary part is correlated with the summation prescription for the IR renormalon divergence in such a way, that the final answer for $\Pi$ is real and unambiguous. There are two messages to be taken from these considerations: First, perturbation theory "knows" about nonperturbative effects through the singularities of the Borel transform on the positive axis ${ }^{6}$. Second, once these effects are identified, they yield strong constraints for the nature of the IR renormalon singularities [34-36]. Thus, from the absence of a dimension-two condensate in Eq. (2.7) one excludes the existence of a singularity at $t=-1 / \beta_{0}$, which according to Eq. (2.6) would call for a $1 / Q^{2}$ term. In addition, the condensates (and therefore their ambiguity) satisfy renormalization group equations, which determine the $\alpha$ dependence of the ambiguity. This must match the ambiguity in the Borel representation of the perturbative series, thus fixing the nature of the corresponding singularity.

We want to emphasize that the appearance of imaginary parts in exponentially small components added to a divergent series is far more general than the SDE, and is just an example of the so-called Stokes phenomenon [37], which generically arises in asymptotic expansions with fixed-sign divergence. It is important to realize that the Stokes discontinuities are formal: After proper summation of all terms, one obtains an analytic function, and, paradoxically, the Stokes discontinuities occur, because the function, which is represented by the asymptotic expansion, is analytic. It is the most economic way for an asymptotic expansion to keep up with the analyticity of the function over a finite phase range of the expansion parameter.

Miraculously, the formal complexities, which we have just reviewed, have never been an obstacle to the practice of QCD sum rules, where, for instance, the gluon condensate is added with some definite value to a few low-order terms of the perturbative expansion. To understand this better, we observe that, although Eq. (2.7) gives the correct asymptotic expansion of $\Pi$, it is not quite the implementation of Wilson's operator product expansion program. This is not designed to separate perturbative and nonperturbative effects into coefficient functions and matrix elements, respectively, an intrinsically ambiguous procedure. Instead it disentangles the physics on different distance scales. Thus one should introduce the factorization scale $\mu<Q$ properly, i.e. cut out the region $k<\mu$ from the momentum integrations in the Feynman diagrams contributing to the coefficient functions and attribute it to the condensates as a non-universal piece. Although this is extremely awkward in practice-see Refs. [38,39] for illustrative examples-one

\footnotetext{
${ }^{5} \mathrm{~A}$ beautiful illustration of this phenomenon has been given in Ref. [15] within the 1/N expansion of the $O(N)$-nonlinear sigma model. Within this expansion dimensional regularization provides a nonperturbative regularization method. Power divergences are then seen to appear as poles at dimensions depending on the order of perturbation theory. To all orders, they accumulate at $d=4$, forcing the limit $d \rightarrow 4$ to be taken through the upper or lower complex $d$-plane, with a different (and complex) result, depending on how the limit is taken.

${ }^{6}$ Clearly, perturbation theory does not know about all nonperturbative effects. For example, in the finite mass case a quark condensate term, $m\langle\bar{q} q\rangle$, appears in Eq. (2.7), which is not seen as a renormalon, because the operator $\bar{q} q$ can not mix with lower dimensional ones, owing to its transformation properties under chiral symmetry. We shall check this explicitly in Sect. 4.
} 


$$
\begin{aligned}
& D_{\mu \nu}^{A B}(u) \equiv \text { 10000 u } u \text { row }
\end{aligned}
$$

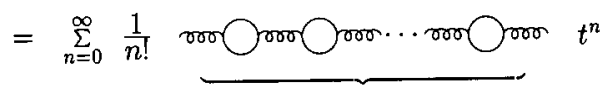

$$
\begin{aligned}
& n \text { bubbles }
\end{aligned}
$$

Fig. 2. Definition of the Borel-transformed gluon propagator. Renormalization of the fermion loops is implied.

may guess conceptually, how Eq. (2.7) is modified. The first perturbative coefficients are not significantly affected, because they are contributed by internal momenta $k \sim Q$. As one progresses towards higher orders, there is a factorially large contribution from momenta $k \sim Q \mathrm{e}^{-n}$, which eventually is eliminated by the constraint $k>\mu$ on the internal integrations. The IR renormalons disappear from all Wilson coefficients. In turn the condensates develop a complicated dependence on $\alpha$. An asymptotic expansion in $\alpha$ reveals the IR renormalon as a perturbative contribution to, e.g., the gluon condensate ${ }^{7}$ :

$$
\begin{aligned}
\left\langle 0\left|\frac{\alpha}{\pi} G G\right| 0\right\rangle(\mu)= & c \mu^{4} \sum_{n}\left(-\frac{\beta_{0}}{2}\right)^{n} n ! n^{-2 \beta_{1} / \beta_{0}^{2}} \alpha(\mu)^{n+1} \\
& +c^{\prime} \mu^{4} \mathrm{e}^{2 /\left(\beta_{0} \alpha(\mu)\right)} \alpha^{2 \beta_{1} / \beta_{0}^{2}}(1+O(\alpha)) .
\end{aligned}
$$

The whole point of the QCD sum rules relies on the fact that this perturbative contribution is small compared to "anomalously" large, genuine nonperturbative effects in the infrared, and can be neglected [40]. From the theory point of view this "rule of discarding the perturbative piece of condensates" remains one of the mysteries of QCD. It could not have been guessed in advance and is justified only by the empirical fact that the sum rules work. In particular, it is far from obvious that the IR renormalons are irrelevant outside the context of the SDE.

Though the existence of renormalons can hardly be doubted on physical grounds, a literal proof does not exist even for the scalar $\Phi^{4}$ theory in four dimensions ${ }^{8}$ due to the failure of continuum field theory in providing a nonperturbative definition of the theory. For this reason, various forms of $1 / N$ expansions have become the state-of-theart approach to renormalons. In QED and, for lack of anything more appropriate, also in QCD, one chooses $1 / N_{f}$ as an expansion parameter, where $N_{f}$ is the number of massless fermions. To organize this expansion, define $a=\alpha N_{f}$ and expand in $1 / N_{f}$ at fixed $a$. In order $1 / N_{f}$, where the renormalons appear first, one has to calculate all diagrams with an arbitrary number of fermion loops inserted into the gluon line of the two-loop diagrams such as in Fig. 1. Since all the dependence on the order in $a$ resides in the number of fermion bubbles, the summation of these diagrams can be taken directly on the gluon propagator, see Fig. 2. The (untruncated) sum of $n$ bubbles is given by

\footnotetext{
${ }^{7}$ The asymptotic behaviour of the perturbative contribution is universal. To connect to the formal position, note that $c^{\prime}$ should formally be considered ambiguous and carries the Stokes discontinuity.

${ }^{8}$ The intrepid reader is referred to Ref. [41], which comes closest to a proof.
} 


$$
D_{\mu \nu, n}^{A B}(k)=i \delta^{A B} \frac{k_{\mu} k_{\nu}-k^{2} g_{\mu \nu}}{\left(k^{2}\right)^{2}}\left(-\pi_{0}\left(k^{2}\right)\right)^{n},
$$

where the Landau gauge has been assumed and renormalization of the fermion bubbles is already understood. Thus

$$
\pi_{0}\left(k^{2}\right)=-\frac{a}{6 \pi}\left(\ln \frac{-k^{2}}{\mu^{2}}+C\right)
$$

with a scheme-dependent finite renormalization constant $C$. In the $\overline{\mathrm{MS}}$ scheme $C=$ $-5 / 3$. It is then easy to find that the Borel-transformed correlation function to order $1 / N_{f}$ is simply obtained by replacing the usual gluon propagator by

$$
D_{\mu \nu}^{A B}(k, u)=\sum_{n=0}^{\infty} \frac{1}{n !} D_{\mu \nu, n}^{A B}(k)\left(\frac{t}{a}\right)^{n}=i \delta^{A B}\left(\frac{\mathrm{e}^{C}}{\mu^{2}}\right)^{-u} \frac{k_{\mu} k_{\nu}-k^{2} g_{\mu \nu}}{\left(-k^{2}\right)^{2+u}} .
$$

We have defined $u \equiv-\beta_{0} t$ with $t$ the Borel parameter. This propagator includes the renormalization of the fermion bubbles, which is equivalent to renormalization of the coupling in the exponent of Eq. (2.5). In this order of the flavour expansion, gluons do not contribute to the $\beta$-function and $\beta_{0}=1 /(6 \pi)$. Unfortunately, we lost the asymptotic freedom property and QCD is identical to QED to this order! In particular, the IR renormalons move to the negative real axis in the Borel plane. Despite its obvious inadequacy to describe the dynamics of QCD, the $1 / N_{f}$ expansion has nonetheless proven successful in detecting the position of renormalons, once we substitute for $\beta_{0}$ its full value $\beta_{0}=1 /(6 \pi)-11 /\left(4 \pi N_{f}\right)$. The reason is of course the intimate relation of renormalons to the scale dependence of the effective coupling. Thus, tracing the fermionic contribution to the $\beta$-function, we get the remaining part-i.e. the gluon and ghost bubbles and whatever else is needed to restore gauge invariance-for free. As an illustration consider the remarkably simple expression for the Borel transform of the correlation function of two vector currents to order $1 / N_{f}[42]^{9}$ (see also Ref. [43]):

$$
\tilde{\Pi}\left(\frac{Q^{2}}{\mu^{2}}, u\right)=-\frac{8}{3 \pi^{3} N_{f}}\left(\frac{Q^{2}}{\mu^{2}} \mathrm{e}^{C}\right)^{-u} \frac{1}{1-\left(\underline{1-u)^{2}}\right.} \sum_{k=2}^{\infty} \frac{(-1)^{k} k}{\underline{\left(k^{2}-(1-u)^{2}\right)^{2}}}
$$

It exhibits the expected UV renormalons at negative integers (the singularity at $u=0$ must be killed by renormalization or by taking one derivative with respect to $Q^{2}$ ) and the IR renormalons at $u=2,3, \ldots$ As required there is no IR renormalon at $u=1$, i.e. $t=-1 / \beta_{0}$, which would correspond to a dimension- 2 operator in the SDE, and the IR renormalon at $t=-2 / \beta_{0}$ can be shown to be a simple pole as a consequence of the vanishing one-loop anomalous dimension of the gluon operator $\alpha / \pi G G[32,42]$. The $1 / N_{f}$ expansion can not detect all singularities of the Borel transform that should be present in QCD. Instanton singularities produce effects that scale as $\exp \left(-4 \pi N_{f} / a\right)$ and will not be seen to any order in $1 / N_{f}$. As far as renormalons are concerned, however,

\footnotetext{
${ }^{9}$ Compared to Ref. [42], the sign in the definition of $u$ has been changed and the overall coefficient adjusted to the QCD case.
} 
all present knowledge supports the assumption that the $1 / N_{f}$ expansion is relevant, provided we substitute $\beta_{0}$ by its full value.

We will employ the $1 / N_{f}$ expansion in the following sections because of its transparency in displaying directly the singularities in the Borel plane, but wish to stress again that this expansion does not contain more information than what can already be extracted from an asymptotic expansion of the Feynman integrands of the low-order diagrams. Indeed, this is just the way to obtain the coefficient functions of higher dimensional operators in the SDE.

\section{The heavy quark expansion: Matching to all orders}

The starting point for HQET, which may be borrowed from nonrelativistic QED, is that the heavy quark spinor splits into a large and a small component, when the heavy quark is almost on-shell. One therefore introduces an effective heavy quark field

$$
h_{v}(x)=\frac{1+\not}{2} \mathrm{e}^{i m_{Q}(v \cdot x)} Q(x)
$$

by projecting on the large component and removing a phase. $v$ is the four-velocity of the heavy quark and $m_{Q}$ is usually referred to as the "heavy quark mass". In this way one arrives at the effective Lagrangian Eq. (1.1). The effective propagator reads

$$
\frac{1+\not}{2} \frac{i}{v \cdot k}
$$

and the quark-gluon vertex is $-i g v_{\mu} T_{a b}^{A}$ (provided one multiplies by $\left(1+\varphi^{\prime}\right) / 2$ for each external heavy quark line), which reveals immediately the flavour and spin independence of the effective theory.

In the following subsections we study in detail the heavy quark expansion of the inverse propagator in QCD to all orders in perturbation theory, its matching onto the HQET, the pole mass of the heavy quark in QCD and the role of the expansion parameter $m_{Q}$. As it turns out, the inverse propagator is not only the simplest, but also the most instructive object to consider. We consider the theory with one heavy and $N_{f}$ massless quarks and expand in $1 / N_{f}$.

\section{I. The self-energy of a heavy quark}

The full propagator in the effective theory can be written as

$$
\frac{1+\not b}{2} i S_{\mathrm{eff}}(v k), \quad S_{\mathrm{eff}}^{-1}(v k) \equiv v k-\Sigma_{\mathrm{eff}}(v k) .
$$

The Borel transform of the self-energy is obtained from the diagram depicted in Fig. 3, where the gluon line represents the summation over an arbitrary number of renormalized fermion bubbles as explained in Sect. 2. Using Eq. (2.11), we are left with a single integration over the gluon momentum with the result $\left(C_{\mathrm{F}}=4 / 3\right)$ 


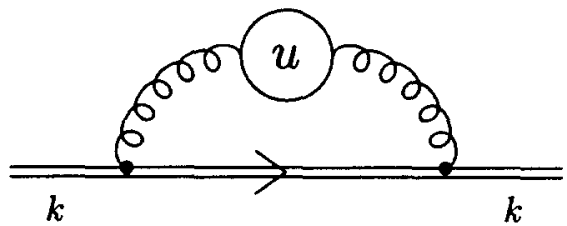

Fig. 3. Diagram for the Borel transform of the self-energy of a heavy quark in HQET.

$$
\tilde{\Sigma}_{\text {eff }}^{\text {part.ren }}(v k, u)=\frac{C_{\mathrm{F}}}{4 \pi N_{f}} v k\left(-\frac{2 v k}{\mu}\right)^{-2 u} \mathrm{e}^{-u C}(-6) \frac{\Gamma(-1+2 u) \Gamma(1-u)}{\Gamma(2+u)} .
$$

All calculations have been performed in dimensional regularization. It turns out that at generic $u$ the result is finite and one can actually put $d=4$ as done in Eq. (3.4). The only renormalization that has still to be done is to account for the overall subtraction of the whole diagram. As shown in Appendix A, this simply amounts to subtracting the pole of the Borel transform at $u=0$ and Eq. (3.4) is corrected to

$$
\tilde{\Sigma}_{\mathrm{eff}}(v k, u)=\tilde{\Sigma}_{\mathrm{eff}}^{\text {part.ren }}(v k, u)+\frac{C_{\mathrm{F}}}{4 \pi N_{f}} v k\left(-\frac{3}{u}+R_{\Sigma_{\mathrm{eff}}}(u)\right) .
$$

The function $R_{\Sigma_{\text {eff }}}(u)$ is entire in the Borel plane, if a renormalization scheme with analytic counterterms is chosen (such as $\overline{\mathrm{MS}}$ ) and can be neglected in the discussion of singularities. More on the issue of scheme dependence can be found in Appendix A. From the definition of the Borel transform in Eq. (2.4) one can read off that the coefficient $p_{n}$ of the perturbative expansion of the self-energy can be recovered from an expansion of the Borel transform in $u$. More precisely, to obtain the coefficient of $a^{n+1}$ in the expansion in the coupling, one has to take $n$ derivatives at $u=0$ :

$$
p_{n}\left(\frac{v k}{\mu}\right)=\left.\left(-\beta_{0}\right)^{n} \frac{\mathrm{d}^{n}}{\mathrm{~d} u^{n}} \tilde{\Sigma}_{\mathrm{eff}}(v k, u)\right|_{u=0} .
$$

In particular, the large- $n$ behaviour is dominated by the pole closest to the origin $u=0$ of the Borel plane.

Let us now scrutinize the singularities of $\tilde{\Sigma}_{\text {eff }}(v k, u)$. We find IR renormalons at positive integer $u$ (i.e. $t=-n / \beta_{0}$ on the positive Borel axis ${ }^{10}$ ) and UV renormalons at $u=1 / 2,-1 / 2,-1,-3 / 2,-5 / 2, \ldots$. To ascertain the UV or IR nature of a given singularity, one either has to inspect the diagram explicitly or to observe the general rule that whenever $u$ occurs with a positive sign in the argument of the Gamma-function in the numerator of a Borel transform, it is UV and with a negative sign it is IR. We are hardly surprised to find IR renormalons on the positive axis, since the effective theory must coincide with QCD in the infrared. The disturbing novelty is an ultraviolet renormalon at positive $u=1 / 2$, which is not Borel-summable and indicates an intrinsic nonperturbative ambiguity of HQET that can not be remedied by any nonperturbative

\footnotetext{
${ }^{10}$ As mentioned previously, we always abstract from the $1 / N_{f}$ expansion and restore the full $\beta_{0}$, which is negative.
} 
effect. The Lagrangian Eq. (1.1) as it stands must therefore be abandoned as a sensible quantum field theory beyond perturbation theory.

It is easy to clarify the origin of this UV-renormalon. The first-order correction to the self-energy is proportional to

$$
\int \frac{\mathrm{d}^{4} p}{(2 \pi)^{4}} \frac{1}{p^{2}(v p+v k)},
$$

which is linearly divergent. The emergence of a linear divergence at this point is physically very transparent. A very heavy quark interacts with its environment only as a static, point-like colour source. The self-energy is then simply given by the energy of the Coulomb field of the source, $\alpha(r) / r$, which is linearly divergent for a point-like object. The divergent part must be included into the renormalization of the mass of the source. As a consequence of this linear divergence, the series of UV renormalons starts from $u=1 / 2$, extending to $u=-\infty$, and not from $u=0$ to $u=-\infty$, as usual. Whereas the standard (dimensional) renormalization of logarithmic divergences subtracts the pole at $u=0$, it does not subtract the linear divergences. This procedure is legitimate as long as one stays within perturbation theory, where a distinction between powers and logarithms is meaningful. Beyond perturbation theory the linear divergences can not be ignored. One could therefore think of introducing a physical, dimensionful cutoff $\lambda$. Inevitably, one induces a counterterm $\lambda \bar{h}_{v} h_{v}$, which can not be absorbed into the parameters of the effective Lagrangian, Eq. (1.1). This reasoning suggests that the HQET may be rescued at the price of introducing an additional parameter that appears as a mass term. Note the similarity with massless $\Phi^{4}$ theory in four dimensions. We encounter a similar kind of fine-tuning, which is very familiar from the scalar theory, in HQET, where the natural mass of the effective heavy quark is $m_{Q}$, the UV cutoff of HQET. This will spoil the heavy quark expansion, since the Lagrangian in Eq. (1.1) has been constructed precisely to eliminate the $m_{Q}$ dependence. To avoid this problem one must impose a renormalization condition on the two-point function that fixes the mass to zero, which is automatically achieved by dimensional renormalization. This does not prevent the appearance of a mass term beyond perturbation theory and, indeed, this is what occurs automatically, when the heavy quark limit is constructed with an expansion parameter which is well defined beyond perturbation theory.

\subsection{Renormalon singularities in the pole mass}

To explain our previous assertion, we digress in this subsection from HQET and deal with the pole mass in QCD. To this end, consider the self-energy of a massive quark. The full propagator is defined by

$$
\begin{aligned}
i S(p, m) & =\frac{i}{\not p-m-\Sigma(p, m)}, \\
\Sigma(p, m) & =m \Sigma_{1}\left(p^{2}, m\right)+(\not p-m) \Sigma_{2}\left(p^{2}, m\right) .
\end{aligned}
$$

The diagram analogous to the one in Fig. 3 but with a quark of finite mass yields the Borel transforms: 


$$
\begin{aligned}
\tilde{\Sigma}_{1}\left(p^{2}, m, u\right)= & \frac{C_{\mathrm{F}}}{4 \pi N_{f}}\left(\frac{m^{2}}{\mu^{2}}\right)^{-u} \mathrm{e}^{-u C} 3 \Gamma(1-u) \Gamma(u)_{2} F_{1}\left(u, 1+u, 2 ; \frac{p^{2}}{m^{2}}\right) \\
& +\tilde{\Sigma}_{2}\left(p^{2}, m, u\right)+\frac{C_{\mathrm{F}}}{4 \pi N_{f}}\left(-\frac{3}{u}+R_{\Sigma_{1}}(u)-R_{\Sigma_{2}}(u)\right), \\
\tilde{\Sigma}_{2}\left(p^{2}, m, u\right)= & \frac{C_{\mathrm{F}}}{4 \pi N_{f}}\left(\frac{m^{2}}{\mu^{2}}\right)^{-u} \mathrm{e}^{-u C}\left(-\frac{3}{2}\right) u \Gamma(1-u) \Gamma(u)_{2} F_{1}\left(u, 2+u, 3 ; \frac{p^{2}}{m^{2}}\right) \\
& +\frac{C_{\mathrm{F}}}{4 \pi N_{f}} R_{\Sigma_{2}}(u) .
\end{aligned}
$$

Here $m$ denotes the renormalized mass (in the scheme specified by $C$ and the functions $R_{\Sigma_{1}}(u)$ and $R_{\Sigma_{2}}(u)$ ) at the normalization point $\mu$ and ${ }_{2} F_{1}$ is the hypergeometric function. Let us pause for a glance at the singularities of the self-energy. If the potential singular point $p^{2}=m^{2}$ of the hypergeometric function is avoided, the UV renormalons occur at negative integers and the IR renormalons at positive integers, just as expected in QCD from the considerations of Sect. 2. The IR renormalon at $u=1$ is not in conflict with the short-distance expansion, which for the inverse propagator contains gauge-variant operators of dimension two like $A_{\mu} A_{\mu}$, where $A_{\mu}$ is the gluon field.

Next we move to the pole mass, which not only is the key quantity in the derivation of the HQET, but also has a considerable interest in itself, as it appears in many phenomenological applications. Then it is important to keep in mind that the concept of a pole mass has no natural extension beyond perturbation theory ${ }^{11}$. Thus we have to find the solution to

$$
\not p-m-\left.\Sigma(p, m)\right|_{p^{2}=m_{\mathrm{pole}}^{2}}=0
$$

in the form of a series expansion

$$
m_{\text {pole }}\left(\frac{m}{\mu}, a\right)=m\left(1+\sum_{n=0}^{\infty} r_{n}\left(\frac{m}{\mu}\right) a^{n+1}\right) \text {. }
$$

Keeping in mind that the self-energy is of order $1 / N_{f}$, we find

$$
m_{\text {pole }}=m\left(1+\Sigma_{1}\left(m_{\text {pole }}^{2}, m\right)+O\left(\frac{1}{N_{f}^{2}}\right)\right) \text {. }
$$

This is still a complicated implicit equation for $m_{\text {pole }}$. A crucial simplification arises from the observation that $m_{\text {pole }}=m+O\left(1 / N_{f}\right)$, which eliminates $m_{\text {pole }}$ from the r.h.s. to order $1 / N_{f}$. Taking the Borel transform of Eq. (3.11) and the explicit expression Eq. (3.9) for $\Sigma_{1}$, we obtain ${ }^{12}$

\footnotetext{
1 There might still be a pole in the propagator, when it is defined in a nonperturbative way. Corresponding to a coloured object, it is however alien to our world.

${ }^{12}$ If a constant term is present, it is useful to include it into the Borel transform with a $\delta$-function, which preserves the form of the inverse Borel transform, Eq. (2.5).
} 


$$
\begin{aligned}
& \tilde{m}_{\text {pole }}\left(\frac{m}{\mu}, u\right) \\
& =m\left(\delta(u)+\frac{G_{\mathrm{F}}}{4 \pi N_{f}}\left[\left(\frac{m^{2}}{\mu^{2}}\right)^{-u} \mathrm{e}^{-u C} 6(1-u) \frac{\Gamma(u) \Gamma(1-2 u)}{\Gamma(3-u)}-\frac{3}{u}+R_{\Sigma_{1}}(u)\right]\right) .
\end{aligned}
$$

The scheme dependence residing in $m$ cancels the scheme dependence of the expression in brackets up to terms of order $1 / N_{f}^{2}$, and $m_{\text {pole }}$ proves to be scheme-invariant, as it must be. In the $\overline{\mathrm{MS}}$ scheme one finds (following the procedure of Appendix A) $R_{\Sigma_{1}}(u)=-5 / 2+35 u / 24+O\left(u^{2}\right)$ and

$$
r_{0}^{\overline{\mathrm{MS}}}\left(\frac{m}{\mu}\right)=m^{-1} \times \tilde{m}_{\mathrm{pole}}\left(\frac{m_{\overline{\mathrm{MS}}}}{\mu}, u=0\right)=\frac{C_{\mathrm{F}}}{4 \pi N_{f}}\left[4+3 \ln \frac{\mu^{2}}{m_{\overline{\mathrm{MS}}}^{2}}\right]
$$

reproduces the well-known relation between the pole mass and the $\overline{\mathrm{MS}}$ mass to lowest order ( $r_{0}$ is the coefficient of $a=\alpha N_{f}$ ).

It is seen immediately from Eq. (3.13) that the on-shell limit created new singularities in the Borel transform! The pole mass has an infrared renormalon at $u=1 / 2$, implying a stronger divergence of the series, Eq. (3.11), than for the expansion of the self-energy at the non-singular points $p^{2} \neq m^{2}$. Without any reference to HQET, this tells us that the pole mass can only be defined up to terms of order $\Lambda_{\mathrm{QCD}}$, unless some ad hoc definition is employed ${ }^{13}$. To make this precise, one may attempt Borel summation and take half the difference of the values obtained from the contour prescription above and below the IR renormalon pole as a measure of the inherent uncertainty of the pole mass,

$$
\delta m_{\text {pole }}=\left|\frac{1}{2} \int_{C^{\prime}} \mathrm{d} t \mathrm{e}^{-t / a(\mu)} \tilde{m}_{\text {pole }}\left(\frac{m}{\mu},-\beta_{0} t\right)\right|,
$$

where the contour $C^{\prime}$ wraps around the positive real axis with the origin excluded. This results in

$$
\begin{aligned}
\delta m_{\text {pole }} & =\frac{\underline{C_{\mathrm{F}}}}{2 N_{f}\left|\beta_{0}\right|} \mathrm{e}^{-C / 2} m(\mu=m) \exp \left(\frac{\underline{1}}{2 N_{f} \beta_{0} \alpha(m)}\right) \\
= & \frac{C_{\mathrm{F}}}{2 N_{f}\left|\beta_{0}\right|} \mathrm{e}^{-C / 2} \Lambda_{\mathrm{QCD}}\left(\ln \frac{m^{2}}{\Lambda_{\mathrm{QCD}}^{2}}\right)^{\beta_{1} /\left(2 \beta_{0}^{2}\right)},
\end{aligned}
$$

where $\beta_{1}=-1 /\left(4 \pi N_{f}\right)^{2} \times\left(102-38 N_{f} / 3\right)$ is the second coefficient of the $\beta$-function (for the rescaled coupling $a=\alpha N_{f}$ ) and we have indicated the renormalization point explicitly. Note that $\mathrm{e}^{-C / 2} \Lambda_{\mathrm{QCD}}$ is scheme-independent $[45,46]$ and the remaining scheme dependence is suppressed by $1 / \ln \left(\mathrm{m}^{2} / \Lambda_{\mathrm{QCD}}^{2}\right)$. An alternative (but scheme-dependent) estimate of $\delta m_{\text {pole }}$ can be obtained from the minimal term of the perturbative expansion

\footnotetext{
${ }^{13} \mathrm{Or}$, to make contact with one of our previous footnotes: The pole of the nonperturbatively defined propagator can be obtained from $\mathrm{Eq}$. (3.11) only by adding terms proportional to $A_{\mathrm{QCD}}$.
} 
and differs from the above by a factor $\left(4 N_{f}\left|\beta_{0}\right| \alpha(m) / \pi\right)^{1 / 2} \approx 0.5$. For a numerical estimate we use an average and obtain

$$
\delta m_{\text {pole }} \approx(170-280) \mathrm{MeV} .
$$

The numerical values are given for the bottom quark and four light flavours. We have varied $\Lambda_{\mathrm{QCD}} \approx(200-300) \mathrm{MeV}$ and $m_{b}\left(m_{b}\right) \approx(4.5-5.3) \mathrm{GeV}$ [44]. We emphasize that this is a crude numerical estimate for three reasons: First, the ambiguity of the Borel sum or the minimal term of the series can only give an indication of the size of the expected nonperturbative effects. Second, the numerical coefficient receives corrections of order $1 / N_{f}^{2}$. Third, the $1 / N_{f}$ expansion does not provide us with the correct nature of the IR renormalon singularity in general-e.g., to all orders in $1 / N_{f}$ one expects the pole to turn into a branch point. Therefore we do not control factors of $\alpha\left(m_{b}\right)$ on the r.h.s. of Eq. (3.17), which can produce a substantial change. Nevertheless, the range of values quoted in Eq. (3.17) should give the right order-of-magnitude estimate for the ambiguities inherent to the concept of the pole mass. The most important, but maybe not too surprising statement [17], is that this ambiguity is of order $\Lambda_{\mathrm{QCD}}$ and not, say, $A_{\mathrm{QCD}}^{2} / m$.

There are a number of simple conclusions to be drawn from the presence of the IR renormalons in the pole mass which still warrant a discussion. A matter of direct relevance is the calculation of total inclusive widths of $B$-hadrons, which is receiving a lot of attention in the literature. Within perturbation theory the total decay widths are given simply by the total widths for the free quark decay, expressions for which can be taken over from QED studies of the muon decay

$$
\Gamma\left(B \rightarrow X_{q} l \bar{\nu}_{l}\right)=\frac{G_{\mathrm{E}}^{2} \mid V_{b} q_{m}^{2} m_{\text {pole }}^{5}}{192 \pi^{3}}(1+\text { perturbative series }) .
$$

The result of principal interest, which triggered all later discussions, is the observation [22] that the leading nonperturbative effects in the total widths are expressed in terms of the expectation values of dimension five operators of the kinetic energy and the chromomagnetic interaction, and are down by two powers of the $b$-quark mass compared to the perturbative contribution. Within the operator product expansion there is no way to obtain corrections of order $1 / m_{b}$, and it is widely believed (see, e.g., Ref. [23]) that perturbation theory is accurate to $1 / \mathrm{m}_{b}^{2}$ accuracy for the total widths, provided the mass parameter which factors Eq. (3.18) coincides with the pole $b$-quark mass. Moreover, the $1 / m_{b}^{2}$ corrections prove to be quite small. In this situation it is appealing to try to determine the pole mass from the experimental data on the total widths, a task undertaken for instance in Ref. [24].

The presence of the IR renormalon in the pole mass invalidates this program. It implies that the ambiguity in the perturbative series defining the pole mass inevitably induces an uncertainty of order $\Lambda_{\mathrm{QCD}} / m_{b}$ for the decay widths. The lesson which should be learnt from the operator product expansion approach of Ref. [22] is that the difference in the total decay widths of different $B$-hadrons is a $O\left(1 / \mathrm{m}_{b}^{2}\right)$ effect, while the question of the absolute normalization is left open. In fact, one could hope that the uncertainties in the summation of the perturbative series in Eq. (3.18) compensates exactly the 
uncertainties in the pole mass, rendering the perturbative prediction unambiguous (up to $1 / m_{b}^{2}$ accuracy) when expressed in terms of the running renormalized quark mass at the scale $m_{b}{ }^{14}$. This question deserves further study.

Thus, the data on the total decay widths can not be used to determine the pole $b$ quark mass, but rather can provide one more definition of it (using the truncated series in Eq. (3.18) ). In this respect, this definition is as good as any other phenomenological definition, e.g. coming from the studies of $B$-mesons or mesons of the $r$ family in the framework of the QCD sum rules [25-27]. The existing estimates for the $b$-quark mass span the range $m_{b}=4.55-4.85 \mathrm{GeV}$, and there has been much debate on which of these values should be preferred. In view of Eq. (3.17) a difference of $\delta m_{\text {pole }} \approx$ few hundred $\mathrm{MeV}$ can easily be attributed to the ambiguity of the definition of a quantity, called "pole mass", beyond perturbation theory. Thus, any claim for $m_{\text {pole }}$ with better accuracy should be considered as hazardous, unless the precise meaning of this quantity is specified.

\subsection{Matching and the residual mass term}

The self-energy of a massive quark contains powers of logarithms of the type $\ln \left(\mathrm{m}^{2}-\right.$ $\left.p^{2}\right) / m^{2}$ (times factors of $\left(m^{2}-p^{2}\right) / m^{2}$ ), which are large, when the quark is heavy and near mass shell, $p^{2}-m^{2} \approx m \Lambda_{\mathrm{QCD}}$. HQET is designed to deal with these large logarithms. To this end, one introduces a factorization scale $\mu$ and writes

$$
\ln \frac{p^{2}-m^{2}}{m^{2}}=\ln \frac{p^{2}-m^{2}}{m \mu}+\ln \frac{\mu}{m} .
$$

The first logarithm is small near mass shell, when $\mu \approx \Lambda_{\mathrm{QCD}}$ is taken and the machinery of renormalization group techniques can then be applied to sum the large logarithms of the type $\ln (m / \mu)$. Remarkably, this factorization can be achieved for the Borel transforms, Eq. (3.9), using an identity that relates hypergeometric functions with argument $z$ and $1-z$. We obtain

$$
\begin{aligned}
\tilde{\Sigma}_{1}\left(p^{2}, m, u\right)=\frac{C_{\mathrm{F}}}{4 \pi N_{f}} \mathrm{e}^{-u C} 3\left\{\left(\frac{m^{2}}{\mu^{2}}\right)^{-u} \frac{\Gamma(u) \Gamma(1-2 u)}{\Gamma(2-u)}{ }_{2} F_{1}\left(u, 1+u, 2 u ; 1-\frac{p^{2}}{m^{2}}\right)\right. \\
+\left(\frac{m^{2}-p^{2}}{m^{2}}\right)\left(\frac{m^{2}-p^{2}}{m \mu}\right)^{-2 u} \\
\left.\quad \times \frac{\Gamma(1-u) \Gamma(-1+2 u)}{\Gamma(1+u)}{ }_{2} F_{1}\left(2-u, 1-u, 2-2 u ; 1-\frac{p^{2}}{m^{2}}\right)\right\} \\
+\tilde{\Sigma}_{2}\left(p^{2}, m, u\right)+\frac{C_{\mathrm{F}}}{4 \pi N_{f}}\left(-\frac{3}{u}+R_{\Sigma_{1}}(u)-R_{\Sigma_{2}}(u)\right),
\end{aligned}
$$

\footnotetext{
14 V.B. is grateful to N.G. Uraltsev for a discussion of this point. We understand that a detailed study of this issue will be presented in Ref. [47], and we gratefully acknowledge receiving a preliminary version of this paper prior to its publication.
} 


$$
\begin{aligned}
\tilde{\Sigma}_{2}\left(p^{2}, m, u\right)= & \frac{C_{\mathrm{F}}}{4 \pi N_{f}} \mathrm{e}^{-u C}(-3 u) \\
& \quad \times\left\{\left(\frac{m^{2}}{\mu^{2}}\right)^{-u} \frac{\Gamma(u) \Gamma(1-2 u)}{\Gamma(3-u)}{ }_{2} F_{1}\left(u, 2+u, 2 u ; 1-\frac{p^{2}}{m^{2}}\right)\right. \\
& +\left(\frac{m^{2}-p^{2}}{m^{2}}\right)\left(\frac{m^{2}-p^{2}}{m \mu}\right)^{-2 u} \\
& \left.\times \frac{\Gamma(1-u) \Gamma(-1+2 u)}{\Gamma(2+u)}{ }_{2} F_{1}\left(3-u, 1-u, 2-2 u ; 1-\frac{p^{2}}{m^{2}}\right)\right\} \\
& +\frac{C_{\mathrm{F}}}{4 \pi N_{f}} R_{\Sigma_{2}}(u) .
\end{aligned}
$$

In the heavy quark limit $1-p^{2} / m^{2} \approx \Lambda_{\mathrm{QCD}} / m$ (provided $m$ is chosen judiciously, see below) and the series expansion of the hypergeometric function realizes directly the heavy quark expansion. At each order, expansion of the Borel transform in $u$ produces two series containing logarithms of either $m^{2} / \mu^{2}$ or $\left(p^{2}-m^{2}\right) /(m \mu)$ only, thus completing the factorization to all orders in the heavy quark expansion and to all orders in perturbation theory in $a$ (but to leading order in $1 / N_{f}$ ). Before we can construct the matching explicitly, we have to discuss the choice of the expansion parameter.

In a heavy meson most of its momentum $p$ is carried by the heavy quark, thus write $p=m_{Q} v+k$. Fixing the velocity $v$ of the heavy quark (thereby selecting a sector in the Hilbert space of the effective theory once and forever), we are still left with some freedom to choose $m_{Q}$. We do not want the residual momentum $k$ to scale with the heavy mass, so intuitively we guess that $m_{Q}$ should be a "physical" mass. In perturbation theory, it does not matter, whether we take the pole mass or the meson mass, but if we want to do better and account for terms of order $\Lambda_{\mathrm{QCD}} / m_{Q}$ consistently, a precise definition of $m_{Q}$ must be given. For the time being, we satisfy ourselves with the observation, that after this is done, we could expand $m_{Q}$ in a double series in the coupling and $\Lambda_{\mathrm{QCD}} / m$, where $m$ is the renormalized mass, of the form

$$
m_{Q}=m\left(1+\sum_{n} s_{n} a(m)^{n+1}\right)-\delta m+O\left(\frac{\Lambda_{\mathrm{QCD}}^{2}}{m}\right) .
$$

This fixes the parameter $m_{Q}$ once and forever and different choices of $m_{Q}$ define different heavy mass expansions. We call

$$
\delta m=C m \mathrm{e}^{1 /\left(2 \beta_{0} \alpha(m)\right)} \alpha(m)^{h^{\prime}}(1+O(\alpha))=C \Lambda_{\mathrm{QCD}} \alpha(m)^{b}(1+O(\alpha))
$$

the residual mass term and $C, b\left(b^{\prime}\right)$ are constants that depend on the definition of $m_{Q}$ (in particular, they could be zero) ${ }^{15}$. With these definitions at hand, we can continue and expand the self-energies, given in Eq. (3.20), in $k / m_{Q}$. To be precise, we will consider

\footnotetext{
${ }^{15}$ Taking $\delta m$ to be of order $\Lambda_{\mathrm{QCD}}$ anticipates $\mathrm{Eq}$. (3.28). Here it is only important to note that, if $\delta m$ is non-zero, it is exponentially small in $\alpha$.
} 
the inverse propagator, see Eq. (3.8), sandwiched between two projectors $(1+\not p) / 2$, and define

$$
\frac{1+\not}{2} S_{P}^{-1}\left(v k, m_{Q}\right)=\frac{1+\not \phi}{2} S^{-1}(p, m) \frac{1+\not}{2} .
$$

After a little algebra, we arrive at the following expression for the Borel transform of the inverse propagator:

$$
\begin{aligned}
& \tilde{S}_{P}^{-1}\left(v k, m_{Q}, u\right)=\tilde{m}_{Q}\left(\frac{m}{\mu}, u\right) \\
& -m\left(\delta(u)+\frac{C_{\mathrm{F}}}{4 \pi N_{f}}\left[\left(\frac{m_{Q}^{2}}{\mu^{2}}\right)^{-u} \mathrm{e}^{-u C} 6(1-u) \frac{\Gamma(u) \Gamma(1-2 u)}{\Gamma(3-u)}-\frac{3}{u}+R_{\Sigma_{1}}(u)\right]\right) \\
& +v k\left[\delta(u)-\frac{C_{\mathrm{F}}}{4 \pi N_{f}} \mathrm{e}^{-u C}\left\{\left(\frac{m_{Q}^{2}}{\mu^{2}}\right)^{-u} 6\left(-1+u^{2}\right) \frac{\Gamma(u) \Gamma(1-2 u)}{\Gamma(3-u)}\right.\right. \\
& \left.\left.+\left(-\frac{2 v k}{\mu}\right)^{-2 u}(-6) \frac{\Gamma(1-u) \Gamma(-1+2 u)}{\Gamma(2+u)}\right\}-\frac{C_{\mathrm{F}}}{4 \pi N_{f}} R_{\Sigma_{2}}(u)\right] \\
& \quad+O\left(\frac{(v k)^{2}}{m_{Q}}, \frac{1}{N_{f}^{2}}\right) \\
& \equiv \tilde{m}_{Q}\left(\frac{m}{\mu}, u\right)-\tilde{m}_{\mathrm{pole}}\left(\frac{m}{\mu}, u\right)+\tilde{C}\left(\frac{m_{Q}}{\mu}, u\right) \star \tilde{S}_{\mathrm{eff}}^{-1}(v k, u)+O\left(\frac{(v k)^{2}}{m_{Q}}, \frac{1}{N_{f}^{2}}\right)
\end{aligned}
$$

Here

$$
\begin{aligned}
\tilde{C}\left(\frac{m_{Q}}{\mu}, u\right)= & \delta(u)-\frac{C_{\mathrm{F}}}{4 \bar{\pi} N_{f}} \mathrm{e}^{-u C}\left(\frac{m^{2}}{\mu^{2}}\right)^{-u} 6\left(-1+u^{2}\right) \frac{\Gamma(u) \Gamma(1-2 u)}{\Gamma(3-u)}, \\
& +\frac{C_{\mathrm{F}}}{4 \pi N_{f}}\left(-\frac{3}{u}+R_{\Sigma_{\mathrm{eff}}}(u)-R_{\Sigma_{2}}(u)\right) \\
\tilde{S}_{\mathrm{eff}}^{-1}(v k, u)= & v k \delta(u)-\tilde{\Sigma}_{\mathrm{eff}}(v k, u),
\end{aligned}
$$

and $\tilde{m}_{\text {pole }}$ and $\tilde{\Sigma}_{\text {eff }}$ have been defined in Eqs. (3.13) and (3.5). The " $\star$ " denotes the convolution product ${ }^{16}$. Finally $\tilde{m}_{Q}$ stands for the Borel transform of the series in Eq. (3.21). The residual mass term is exponentially small in the coupling and therefore not seen in the "perturbative" definition of the Borel transform, which we use throughout this paper. Therefore including possible terms of order $\Lambda_{\mathrm{QCD}}$ from the definition of $m_{Q}$, we recover the inverse propagator through

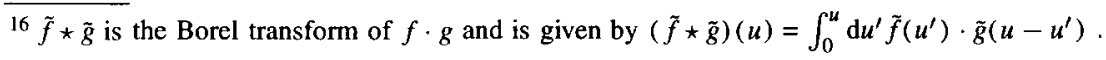




$$
S_{P}^{-1}\left(v k, m_{Q}, a\right)=\int_{0}^{\infty} \mathrm{d} t \mathrm{e}^{-t / a} \tilde{S}_{P}^{-1}\left(v k, m_{Q},-\beta_{0} t\right)-\delta m+O\left(\frac{A^{2}}{m_{Q}}\right) .
$$

Eq. (3.24) is crucial for understanding the structure of renormalon singularities in the heavy quark limit and is worth being discussed in great detail. Assume first that the expansion parameter $m_{Q}$ equals the renormalized mass $m$, i.e. $\tilde{m}_{Q}=m \delta(u)$ and $\delta m=0$. Let us list the following observations:

(1) In perturbation theory $u$ should be considered as infinitesimal and factors like $\left(m_{Q}^{2} / \mu^{2}\right)^{-u}$ turn into a series in $\ln \left(m_{Q}^{2} / \mu^{2}\right)$, when $\tilde{S}_{P}^{-1}$ is expanded in $u$ to yield the perturbative expansion of $S_{P}^{-1}$ in $a$. Eq. (3.24) has a very simple structure: The first two lines scale with $m_{Q}$ and are given by $\tilde{m}_{Q}-\tilde{m}_{\text {pole }}$. The term proportional to $v k$ has a factorized form and can be written as the product of a coefficient function $\tilde{C}$, that contains all the (logarithmic) dependence on $m_{Q}$, and the effective inverse propagator, which is $m_{Q}$-independent. These terms appear as a sum and not as a product in Eq. (3.24), because we neglect terms of order $1 / N_{f}^{2}$, cf. Eq. (3.25). It is evident from Eq. (3.20) that this factorization holds true in higher orders in the $1 / m_{Q}$ expansion, where to order $(v k)^{2} / m_{Q}$ it matches onto the kinetic and magnetic energy contribution to the self-energy of a heavy quark.

(2) The term proportional to $v k$ is finite at $u=0$ as it must be for the renormalized inverse propagator. However, the two terms in curly brackets-corresponding to coefficient function and effective propagator ${ }^{17}$-have poles at $u=0$ separately. Factorization has introduced UV divergences into coefficient functions and matrix elements. By subtracting and adding a term $(-3) / u+R_{\Sigma_{\text {eff }}}(u)$ to the expression in curly brackets as indicated in Eq. (3.25), one chooses a particular factorization scheme. As known from many other examples there is an arbitrariness in the separation of contributions to coefficient functions and matrix elements, which here is represented by the arbitrary function $R_{\Sigma_{\text {eff }}}(u)$. In the language of HQET, a particular factorization scheme corresponds to a particular wave function renormalization of the effective heavy quark field $h_{v}$.

(3) Consider now Eq. (3.24) at finite $u$. In this way we probe the asymptotic behaviour of the perturbative expansion (in $u$ or, equivalently, in $a$ ) and explore the nonperturbative effects which are seen by the renormalon singularities. In view of our previous discussion, we are mainly interested in the point $u=1 / 2$, but the effect of factorization on renormalons is quite general: it introduces new infrared renormalons into the coefficient functions (from $\Gamma(1-2 u)$ ) and ultraviolet renormalons into the matrix elements (from $\Gamma(-1+2 u)$ ), which are not present in the non-expanded inverse propagator. Thus these singularities must cancel each other, just as the singularity at $u=0$ does, and indeed they do, but the cancellation takes place among different orders in the $1 / m_{Q}$ expansion. For example, the IR renormalon in the pole mass at $u=1 / 2$ cancels the UV renormalon at $u=1 / 2$ in $\tilde{S}_{\text {eff }}^{-1}$ and similarly, the singularity in $\tilde{C}$ cancels an UV renormalon in the matrix elements in the next order in $1 / m_{Q}$. At $u=3 / 2$ the

\footnotetext{
${ }^{17}$ In a slight abuse of language, we shall refer to the effective quantities that depend on $v k$ also as "matrix elements".
} 
cancellation takes place over three orders in $1 / m_{Q}$. Of course, the pole at $u=1$ is not fully eliminated, since it is present in $\tilde{S}_{P}^{-1}$ as mentioned previously.

(4) Factorization thus affects the structure of renormalons in a very similar way as we are used to in finite orders of perturbation theory (a small neighbourhood of $u=0$ ) with the difference that the divergences are related over different orders in the expansion parameter $m_{Q}$. It is tempting to introduce a factorization scheme for renormalon poles in general, just as one usually does in perturbation theory, i.e. regarding the "renormalon" pole at $u=0$ in particular. For instance, the replacements

$$
\begin{gathered}
\tilde{m}_{\text {pole }}\left(\frac{m}{\mu}, u\right) \longrightarrow \tilde{m}_{\text {pole }}\left(\frac{m}{\mu}, u\right)+\mu \frac{C_{\mathrm{F}}}{4 \pi N_{f}} \frac{4}{1-2 u}, \\
\tilde{S}_{\text {eff }}^{-1}(v k, u) \longrightarrow \tilde{S}_{\text {eff }}^{-1}(v k, u)+\mu \frac{C_{\mathrm{F}}}{4 \pi N_{f}} \frac{4}{1-2 u}
\end{gathered}
$$

will eliminate the divergence coming from $u=1 / 2$ from the perturbative expansions of the pole mass and the effective self-energy without affecting $\tilde{S}_{P}^{-1}$. Technically, this can be achieved within dimensional regularization by subtraction of the poles at $d=3$. However, such a substitution messes up the $1 / m_{Q}$ expansion, since it introduces powers of the scale $\mu$. In fact, it acts analogously to a hard cutoff in the Feynman integrals, which removes the contribution to the pole mass from the IR region and from the UV region to the self-energy. We recognize that the present discussion of the heavy mass expansion parallels the discussion of the short-distance expansion in Sect. 2 and indeed the renormalons appear for the very same reason, that, e.g. for the coefficient functions, diagrams with a large number of bubbles are dominated by internal momenta smaller than $\mu$. Quantitatively, the crucial difference to the SDE is that the infrared effects appear at order $A_{\mathrm{QCD}} / m_{Q}$ (take $\mu=m_{Q}$ ) and not at order $A_{\mathrm{QCD}}^{4} / Q^{4}$.

After these remarks on the heavy mass expansion in general, we are in the position to consider the construction of HQET. The effective Lagrangian, Eq. (1.1), is independent of $m_{Q}$ and supposed to extract the correct dependence of the Green functions on $m_{Q}$ to leading order. From Eq. (3.24) it follows that this purpose cannot be accomplished with the renormalized mass as the expansion parameter, but one must choose $m_{Q}$ such that it coincides with the pole mass to all orders of perturbation theory,

$$
\tilde{m}_{Q}\left(\frac{m}{\mu}, u\right)=\tilde{m}_{\text {pole }}\left(\frac{m}{\mu}, u\right),
$$

in order to cancel the term that scales with $m_{Q}$. This results in ${ }^{18}$

$$
\tilde{S}_{P}^{-1}\left(v k, m_{Q}, u\right)=\tilde{C}\left(\frac{m_{Q}}{\mu}, u\right) \star \tilde{S}_{\text {eff }}^{-1}(v k, u)+O\left(\frac{(v k)^{2}}{m_{Q}}, \frac{1}{N_{f}^{2}}\right) .
$$

\footnotetext{
${ }^{18}$ It is sometimes understood that the coefficient function for the propagator is unity. This can be achieved by a particular choice of the renormalization scheme in QCD via the function $R_{\Sigma_{2}}(u)$, see Eq. (3.24). Clearly, this requires a mass-dependent scheme with non-analytic counterterms (see Appendix A) and for this reason we refrain from performing this step, when we discuss the singularities of the Borel transform. It is not strictly necessary and will not be important for the following.
} 
The price to pay for the elimination of all terms scaling with $m_{Q}$ in perturbation theory is, however, that the renormalon singularity at $u=1 / 2$ is no longer cancelled. With $\tilde{S}_{P}^{-1}$ of Eq. (3.29) inserted into Eq. (3.26), the Borel integral is no longer well defined up to $u=1$ as it was with the non-expanded inverse propagator (or the choice $m_{Q}=m$ ). We have introduced a spurious renormalon into the construction of HQET, which renders the Borel integral ambiguous by terms of order $A_{\mathrm{QCD}}$ ! This is not a big surprise, because the Borel integral for $m_{Q}$ is itself ambiguous by terms of this order, see Eq. (3.28), and the discussion of the previous subsection. It is now high time to return to the definition of $m_{Q}$, Eq. (3.21).

Once we want to use HQET beyond perturbation theory and include corrections of order $\Lambda_{\mathrm{QCD}} / m_{Q}$ an unambiguous definition of $m_{Q}$ becomes imperative. Let us therefore imagine a summation prescription for the divergent expansion in $\mathrm{Eq}$. (3.21) that defines $m_{Q}$ as a function of $a$ with certain analyticity properties. This fixes the exponentially small in $a$ terms, which are not seen in the perturbative expansion. We can accomplish this with Borel summation, if we add a residual mass term $\delta m$ of order $\Lambda_{\mathrm{QCD}}$ as in Eq. (3.21) and understand that it is formally ambiguous and possibly complex. To explain this, recall that one must give a prescription for the pole in the Borel integral at $u=1 / 2$. The Borel integral will differ whether one chooses to deform the contour into the upper or lower complex plane and the residual mass term must cancel this ambiguity, if $m_{Q}$ itself is unambiguous ${ }^{19}$. This residual mass term is obligatory and its presence in Eq. (3.26) cancels precisely the ambiguity from the spurious pole at $u=1 / 2$ in the Borel sum. In this sense the status of the residual mass term is identical to the status of the condensates in the SDE. Recall that the ambiguity in the definition of condensates compensates the IR renormalons in the perturbative expansion. It is important that $\delta m$ is not a physical quantity. It is defined as a number only after one has fixed a summation prescription for the perturbative expansion of the Green functions in HQET (which, of course, should be done consistently). Apart from this formal analogy, the residual mass term is very different from condensates in the SDE and certainly can not be related to condensates in any way. It has no direct dynamical origin, but it is through this ambiguous residual mass, that HQET remembers that the concept of a "quark on mass-shell" is not physical, even if the quark is very heavy. The modifications of the effective Lagrangian of HQET in the presence of a residual mass term have already been given in Ref.[16]. If we understand the ambiguous nature of $\delta m$, we can in fact copy all expressions given there. In particular, the leading-order Lagrangian, Eq. (1.1), has to be modified in the obvious way

$$
\mathcal{L}_{\text {eff }}=\bar{h}_{v} i v \cdot D h_{v}-\delta m \bar{h}_{v} h_{t}+\mathcal{L}_{\text {light }}
$$

\footnotetext{
${ }^{19}$ This tacitly assumes that an asymptotic expansion of $m_{Q}$ in $a$ can be performed in a sector around the positive real $a$-axis and that the fixed sign divergence for positive $a$ is indeed correlated with a Stokes discontinuity in the exponentially suppressed terms. We are far from proving such a statement, which is, however, an underlying assumption in practically all works, which relate the asymptotic behaviour of perturbation series to "nonperturbative terms" and single out the Borel summation and its extensions to series with fixed sign divergence. It has been verified in model calculations [15], that this gives the correct prescription, but is conjecture beyond.
} 
The appearance of an ambiguous quantity in the Lagrangian might seem peculiar. But this Lagrangian arises as a remnant of QCD. Without fixing a specific summation prescription for the Green functions in QCD before the construction of HQET, the ambiguous residual mass appears in the above Lagrangian to render the Green functions derived from this Lagrangian unambiguous and invariant under different summation prescriptions.

To recognize the implications of an ambiguous residual mass term, define $\bar{A}$ as the difference of the heavy hadron and the heavy quark expansion parameter in the heavy mass limit:

$$
m_{P}-m_{Q}=\bar{\Lambda}+O\left(\frac{1}{m_{Q}}\right) .
$$

By construction, $\bar{A}$ is well defined, but it inherits the arbitrariness inherent in the definition of $m_{Q}$. It has been shown [16] that the matrix elements of HQET depend only on the combination

$$
\bar{\lambda}=\bar{\Lambda}-\delta m,
$$

which is invariant under redefinitions of $m_{Q}$. It turns out to be an important quantity for the parametrization of the matrix elements to order $1 / m_{Q}$. For example, it is the only unknown parameter governing the $1 / m_{Q}$ corrections to the decays of a heavy baryon, where the light quarks are in a spin zero state [48]. We conclude from our above analysis that this quantity is theoretically ambiguous by terms of order $\Lambda_{\mathrm{QCD}}$ and has no physical meaning by itself. The "operational" definition, given in Ref. [16],

$$
\bar{\Lambda}-\delta m=\frac{\left\langle 0\left|\bar{q}(i v \cdot \overleftarrow{D}) \Gamma h_{v}\right| M(v)\right\rangle}{\left\langle 0\left|\bar{q} \Gamma h_{v}\right| M(v)\right\rangle},
$$

where $|M(v)\rangle$ denotes a meson state, $q$ a light quark field and $\Gamma$ a Dirac matrix, is in fact illusive. The matrix element is ambiguous. It can not be directly related to any physical quantity and defining it requires a nonperturbative regularization, which can not avoid the renormalon problem. If $\bar{\lambda}$ were a physical quantity and could be unambiguously determined, this would provide us with a unique nonperturbative definition of a heavy quark mass, $m_{\text {pole, }}$ through Eq. (3.31) as is indeed widely maintained (see e.g. Refs. $[23,29])$. Unfortunately, this is not so. The decay of a $\Lambda_{b}$ baryon [48] may serve as an illustration. The value of $\bar{\lambda}$ enters the form factors of this decay at subleading order in $1 / m_{Q}$. The leading-order form factors receive perturbative corrections with a divergent series expansion which is expected to have a renormalon at $u=1 / 2$. Theoretically, corrections of order $\Lambda_{\mathrm{QCD}} / m_{Q}$ are well defined only after the choice of a summation prescription for this series. Unless this is done, $\bar{\lambda}$ must be ambiguous. Practically, it might still be useful to fit a value for $\bar{\lambda}$ phenomenologically where, again, we appeal to the analogy with the condensates in the SDE. But there can be no rigorous theoretical determination of $\bar{\lambda}$, as has already been observed on physical grounds in Ref. [17], which in fact has triggered our more formal investigation. 


\section{The correlation function of heavy-light currents in HQET}

While the quark propagator has been very useful to gain some insight into the structure of the heavy quark expansion in large orders of perturbation theory, it is not a quantity of particular physical interest. We devote this section to the study of the Borel transform of the correlation function of heavy-light currents in HQET. Its spectral density contains a heavy meson pole and the short-distance expansion of the correlation function provides access to $\bar{\Lambda}$ and the decay constant of the meson through the technique of the QCD sum rules. To be definite, we consider the perturbative expansion to order $1 / N_{f}$ of

$$
\Pi_{5}(\omega)=i \int \mathrm{d}^{4} x \mathrm{e}^{i \omega(v \cdot x)}\left\langle 0\left|T\left\{j_{5}^{\dagger}(x) j_{5}(0)\right\}\right| 0\right\rangle, \quad j_{5}(x)=\bar{h}_{v}(x) i \gamma_{5} q(x) .
$$

The choice of the Dirac matrix turns out to be unimportant, since the pseudoscalar and vector mesons are degenerate to leading order in the $1 / m_{Q}$ expansion. The variable $\omega=v q$ has the meaning of a frequency, and measures the off-shellness of the heavy quark, provided that $m_{Q}$ has been unambiguously defined as explained in the previous section and $q$ is the residual momentum. We will not construct the full matching to QCD as for the inverse propagator, which would require the calculation of the corresponding correlation function in QCD with a massive quark to obtain the coefficient function. $\Pi_{5}(\omega)$ is quadratically divergent and the current product needs an additional subtraction, which is a second-order polynomial in $\omega$. This subtraction can be avoided by taking three derivatives and we shall consider

$$
D(\omega) \equiv \omega \frac{\mathrm{d}^{3} \Pi_{5}(\omega)}{\mathrm{d} \omega^{3}}
$$

in the following.

The SDE of the corresponding correlation function in QCD can be repeated in HQET in a two-step procedure. First, the momenta larger than $m_{Q}$ are integrated out, which results in a series in $1 / m_{Q}$ of correlation functions of operators in the HQET of which $\Pi_{5}(\omega)$ is the first term. Second, the products of effective operators are expanded at short distances, that is $\Lambda_{\mathrm{QCD}} \ll \omega<m_{Q}$. The SDE of $\Pi_{5}(\omega)$ is given by [49]

$$
\begin{aligned}
D\left(\frac{\omega}{\mu}, \alpha(\mu)\right)= & -\frac{3}{\pi^{2}}\left(1+\frac{\alpha(\mu)}{\pi}\left\{\frac{8}{3}+\frac{4 \pi^{2}}{9}-2 \ln \left(-\frac{2 \omega}{\mu}\right)\right\}+O\left(\alpha^{2}\right)\right) \\
& -\frac{3}{\omega^{3}}\langle\bar{q} q\rangle(\mu)\left(1+2 \frac{\alpha(\mu)}{\pi}+O\left(\alpha^{2}\right)\right) \\
& +\frac{15}{4 \omega^{5}}\langle g \bar{q} \sigma G q\rangle(\mu)(1+O(\alpha))+\ldots,
\end{aligned}
$$

where the omitted series of higher dimensional operators starts with four-quark operators. Since the heavy-light current acquires an anomalous dimension in the effective heavy quark theory, the two-loop perturbative correction is now scheme dependent in contrast to the case of vector currents of light quarks, and the above result is given in the $\overline{\mathrm{MS}}$ scheme. Note also that to leading order in the $1 / m_{Q}$ expansion there is no contribution from the gluon condensate. 


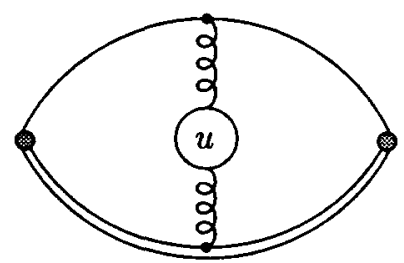

(a)

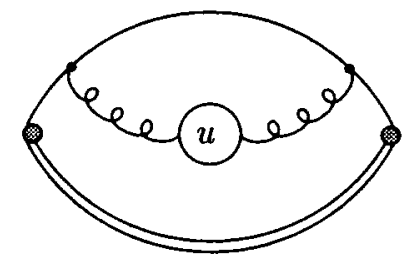

(b)

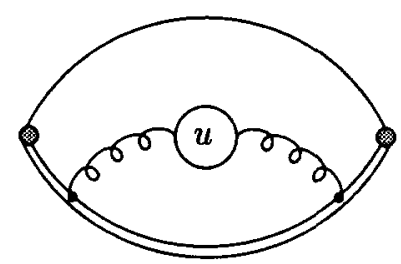

(c)

Fig. 4. Diagrams for the Borel transform of the correlation function of heavy-light currents in HQET. The shaded circle stands for an insertion of the current with momentum $q$, the double line denotes the effective heavy quark propagator.

As familiar by now, to order $1 / N_{f}$ we are interested in the contribution from diagrams with an arbitrary number of light quark loops inserted into the gluon line of the two-loop diagrams. The Borel transform of this class of diagrams can conveniently be computed by inserting the Borel-transformed gluon propagator, Eq. (2.11), see Fig. 4. The remainder is technical. On the one hand the calculation is far less tedious than in the light quark case [42], since the spinor structure simplifies in the heavy quark limit. On the other hand, the correlation function has an anomalous dimension and one looses the Ward identity (which holds in QCD to order $1 / N_{f}$ ), which ensured that all divergences cancel after one subtraction of the correlation function of light quark currents. The correlation function of the effective heavy-light currents needs an explicit renormalization and we refer again to Appendix A, where the procedure is outlined. It turns out that only the diagram (c) in Fig. 4 has a pole at $u=0$, which is eliminated in this way. The most difficult part comes from the non-reducible scalar part of the diagram (a). The details of its computation are given in Appendix B.

The result for the Borel transform of the correlation function is

$$
\begin{aligned}
\tilde{D}\left(\frac{\omega}{\mu}, u\right)= & -\frac{N_{c}}{\pi} \delta(u)+\tilde{D}_{(\mathrm{a})}\left(\frac{\omega}{\mu}, u\right)+\tilde{D}_{(\mathrm{b})}\left(\frac{\omega}{\mu}, u\right) \\
& +\tilde{D}_{(\mathrm{c})}\left(\frac{\omega}{\mu}, u\right)+O\left(\frac{1}{N_{f}^{2}}\right)
\end{aligned}
$$


with the separate contributions (in the Landau gauge) from the three diagrams shown in Fig. 4 given by $\left(N_{c}=3, C_{\mathrm{F}}=4 / 3\right)$

$$
\begin{aligned}
\tilde{D}_{(\mathrm{a})}\left(\frac{\omega}{\mu}, u\right) & =\frac{C_{\mathrm{F}} N_{c}}{4 \pi^{3} N_{f}} \\
\times & {\left[\left(-\frac{2 \omega}{\mu}\right)^{-2 u} \mathrm{e}^{-u C}\{2 u(1-2 u)(2-2 u)[S(4, u)-S(4,1+u)]\right.} \\
& \left.\left.+\frac{\Gamma(-u) \Gamma(1+2 u)}{\Gamma(2+u)}\left[3+5 u-\frac{1+u}{1-2 u}\right]\right\}+R_{(\mathrm{a})}(u)\right], \\
\tilde{D}_{(\mathrm{b})}\left(\frac{\omega}{\mu}, u\right) & =\frac{C_{\mathrm{F}} N_{f}}{4 \pi^{3} N_{f}}\left[\left(-\frac{2 \omega}{\mu}\right)^{-2 u} \mathrm{e}^{-u C} 3 \frac{\Gamma(1-u) \Gamma(1+2 u)}{(2-u) \Gamma(2+u)}+R_{(\mathrm{b})}(u)\right], \\
\tilde{D}_{(\mathrm{c})}\left(\frac{\omega}{\mu}, u\right) & =\frac{C_{\mathrm{F}} N_{c}}{4 \pi^{3} N_{f}}\left[\left(-\frac{2 \omega}{\mu}\right)^{-2 u} \mathrm{e}^{-u C} 6 \frac{\Gamma(1-u) \Gamma(-1+2 u)}{\Gamma(2+u)}+\frac{3}{u}+R_{(\mathrm{c})}(u)\right],
\end{aligned}
$$

where

$$
\begin{aligned}
S(4, \gamma)= & -\Gamma(1-\gamma) \Gamma(2-\gamma) \Gamma(\gamma-1)^{2} \\
& +2 \frac{\Gamma(1-\gamma) \Gamma(2 \gamma-1)}{\Gamma(\gamma-1)} \sum_{n=0}^{\infty} \frac{1}{\gamma-1+n} \frac{\Gamma(2 \gamma-2+n)}{\Gamma(3 \gamma-2+n)} \frac{\Gamma(\gamma-1+n)}{n !},
\end{aligned}
$$

(see Eq. (B.10)). The $R$-functions are scheme dependent and arbitrary in general apart from being non-singular in a neighbourhood of $u=0$. In MS-like schemes, they are entire and their values at $u=0$ can be found to be $R_{(\mathrm{a})}(0)=0, R_{(\mathrm{b})}(0)=-3 / 2$, $R_{(\mathrm{c})}(0)=4$. A check of our result is provided by the value of the Borel transform at $u=0$ (disregarding the $\delta$-function), which must reproduce the two-loop perturbative correction to $D$. With the help of the expansions collected in Appendix B, we find

$$
\begin{aligned}
& \tilde{D}_{(\mathrm{a})}\left(\frac{\omega}{\mu}, 0\right)=\frac{3 C_{\mathrm{F}} N_{c}}{4 \pi^{3} N_{f}}\left\{\frac{4}{3}+\frac{4 \pi^{2}}{9}\right\}, \quad \tilde{D}_{(\mathrm{b})}\left(\frac{\omega}{\mu}, 0\right)=0, \\
& \tilde{D}_{(\mathrm{c})}\left(\frac{\omega}{\mu}, 0\right)=\frac{3 C_{\mathrm{F}} N_{c}}{4 \pi^{3} N_{f}}\left\{-\frac{1}{3}-C-2 \ln \left(-\frac{2 \omega}{\mu}\right)\right\},
\end{aligned}
$$

in agreement with Eq. (4.3) in the $\overline{\mathrm{MS}}$ scheme $(C=-5 / 3)$.

We now turn to the discussion of the renormalon singularities in the Borel plane. A summary of this discussion is presented in Fig. 5, where the Borel plane for the correlation function of heavy-light currents in HQET is compared with the situation for light quarks in QCD.

Infrared renormalons. The IR singularities occur at positive integers starting from $u=3$ and, generically, are double poles. The poles at $u=1$ and $u=2$ are present in every single diagram but cancel in the sum of all three. In general, a condensate of dimension $d$ 


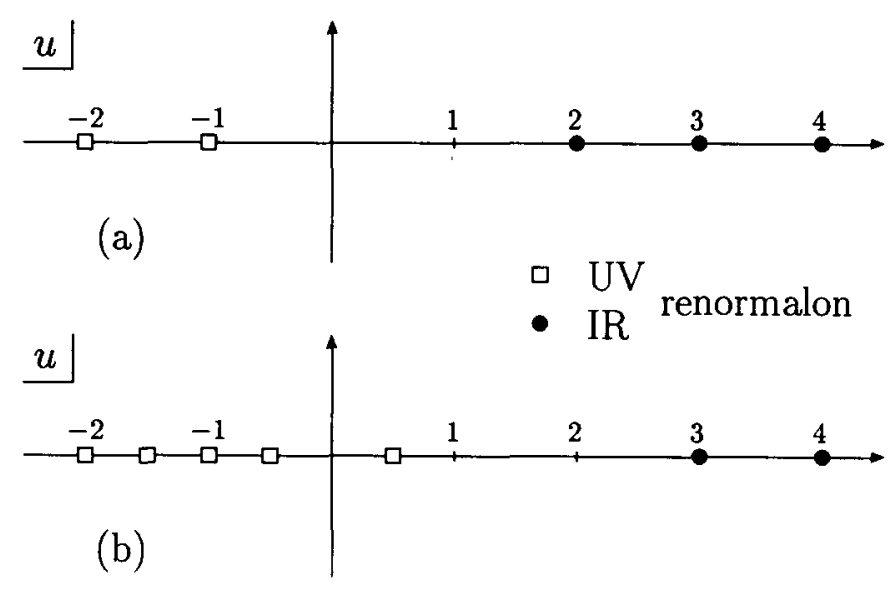

Fig. 5. Singularities in the Borel plane to order $1 / N_{f}$ for (a) the correlation function of vector currents in QCD and (b) the correlation function of heavy-light currents in HQET.

in the SDE can be related to an IR renormalon at $u=d / 2$. By comparison with Eq. (4.3) we find that the quark condensate and the mixed quark-gluon condensate do not produce IR poles in the coefficient function of the unit operator. This is physically clear, because the renormalons originate from a soft gluon line in the diagrams of Fig. 4. More formally, the renormalons are linked to an ambiguity in the definition of the vacuum expectation values of composite operators due to mixing with lower dimensional operators in the sense that the definition of condensates in principle requires a prescription for the sum of all perturbative series that appear in lower dimensional terms. The operator $\bar{q} q$ can not mix with any lower dimensional operator due to its different transformation properties under chiral symmetry ${ }^{20}$ and its vacuum expectation value is unambiguously determined by the pion decay constant [15] through the PCAC relation. The mixed quark-gluon operator $g \bar{q} \sigma G q$ has no such protection, but due to its chiral transformation properties mixes only with $\bar{q} q$. For this reason, the mixed quark gluon condensate is not seen as an IR renormalon in the perturbative expansion, but should be related to an IR renormalon in the Borel transform of the coefficient function of the chiral condensate at $u=1$.

The cancellation of the IR renormalon at $u=2$ can be directly attributed to the absence of the gluon condensate in the SDE to leading order of the $1 / m_{Q}$ expansion. Thus, all IR renormalons in the correlation function are in complete agreement with the SDE. The first singularity at $u=3$ comes from four-quark operators not written in Eq. (4.3). Since there are no IR renormalons at half integers, we conclude that odd dimensional operators do not produce IR poles in the coefficient function of the unit operator, which is again a consequence of chiral symmetry. If we assume that the equation of motion has been used to reduce all operators with covariant derivatives, then odd dimensional gauge-invariant operators must contain $4 k+2(k=0,1, \ldots)$ quark

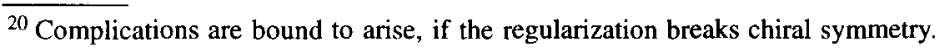


fields and an arbitrary number of gluon field strengths and a chiral-invariant operator with this number of quark fields can not be constructed.

Ultraviolet renormalons. In contrast to the light quark case, UV renormalons appear in the perturbative expansions in HQET at all negative half integers for the simple reason that there is a dimension one parameter $\omega$ available. In addition, one finds a non-Borel-summable UV renormalon at $u=1 / 2$ on the positive axis, which, in fact, stems only from the diagram (c). The pole at $u=1 / 2$ in some terms of $\tilde{D}_{(\text {a) }}$ is spurious and drops out in the full expression. This UV renormalon is a simple pole and can be traced to the insertion of the self-energy of the heavy quark in the diagram (c), which we have investigated in detail in Sect. 3, where the linear divergence of the self-energy has been identified as the cause of this pole. Note that the correlation function $\Pi_{5}(\omega)$ is quadratically divergent and has UV poles at $u=1 / 2$ and $u=1$, which have been eliminated by taking three derivatives. In a similar way the UV renormalon at $u=1 / 2$ is removed from the first derivative $\partial \tilde{\Sigma}_{\text {eff }}(v k) / \partial(v k)$ of the self-energy, but since $\Sigma_{\text {eff }}(v k)$ and not its derivative is inserted into the Green functions of HQET, there is no way to avoid the UV renormalon generated by the linear divergence of the self-energy to pervade to all Green functions in HQET.

The emergence of non-summable singularities on the positive Borel axis signals that perturbation theory is incomplete and points towards either inconsistency of the theory or some nonperturbative phenomenon which in a formal language cures the ambiguities of the Borel integral. For the IR renormalons in HQET this is provided by the condensates just as in QCD and they account for the nonperturbative terms that arise in the SDE of the correlation functions. The UV renormalon at $u=1 / 2$ reminds us of a nonperturbative effect of a very different nature, which can not be attributed to short distances: HQET (without a residual mass term) is an effective theory for a heavy on-shell fermion that does not exist in nature and the binding energy of a quark in a meson is not a physical concept. Indeed, we have seen in Sect. 3 that one must add a residual mass term $\delta m$ to the effective Lagrangian, see Eq. (3.30), which we have omitted in our discussion so far. Since in the $1 / N_{f}$ expansion $\delta m$ counts as $1 / N_{f}$, to order $1 / N_{f}$ the term

$$
D_{\delta m}\left(\frac{\omega}{\mu}, \alpha(\mu)\right)=\frac{N_{c}}{\pi^{2}} \frac{\delta m}{\omega}
$$

has to be added to Eq. (4.3), which cancels the ambiguity of the Borel integral for $D$ due to the UV renormalon. We repeat that the residual mass term is formally of order $\Lambda_{\mathrm{QCD}}$ and serves two purposes: (1) It guarantees that the predictions of HQET are invariant under the choice of the expansion parameter $m_{Q}[16],(2)$ it ensures that the predictions of HQET are invariant under the choice of summation prescription for the UV renormalon divergence of the correlation functions in HQET. In this respect, it acts analogously as the condensate terms in Eq. (4.3) with respect to the IR renormalons. To be precise, one could agree that all series should be summed by a contour of the Borel integral through the upper complex plane, which would fix $\delta m$ with an imaginary part that ensures reality of the correlation functions and defines a particular $m_{Q}$. One can convince oneself that if this is accomplished for the self-energy, then it is automatic for $D$, where it is an important consistency check that the diagrams (a) and (b) do not produce a pole at $u=1 / 2$. Superficially the presence of a residual mass leads to 
new terms of order $\Lambda_{\mathrm{QCD}} / \omega$ in Eq. (4.3), which can not be avoided because of the divergence of perturbation series and which spoil the SDE, where it is assumed that all power corrections can be accounted for by condensates. We shall show below that the power corrections due to the residual mass are organized in a very particular way and can effectively be summed up.

Let us first throw a glance beyond the $1 / N_{f}$ expansion. Consider the class of diagrams, where a second heavy quark self-energy is inserted into the heavy quark line of the diagram (c) of Fig. 4 and with an arbitrary number of fermion loops in any of the two gluon lines. Apart from factors these diagrams can be obtained by squaring the series in $\alpha$, implied by the diagram (c). The Borel transform is given by the convolution of diagram (c) with itself and develops an UV renormalon at $u=1 / 2$ and $u=1$. Obviously, this process can be iterated and the diagrams with a chain of $n$ self-energies produce a Borel transform with UV poles at all half integers up to $n / 2$, which are of course related to $n$ insertions of the residual mass term. We conclude that to all orders in perturbation theory, the UV renormalons proliferate and spread over all half-integers on the negative and positive Borel axis. Opposite to the situation with light quark currents, where the leading order in $1 / N_{f}$ gives a complete picture of the renormalons in the Borel plane (as far as we know), see Fig. 5a, the Borel plane of the correlation function of heavy-light currents in HQET becomes modified to all orders in $1 / N_{f}$. As in QED, there is a series of UV renormalons on the positive axis, but it must be emphasized that their physical origin is so completely different that the common name is hard to justify: In QED, the UV renormalons arise from the logarithmic increase of the effective coupling in the UV region; in HQET all UV renormalons are generated by the linear divergence of the heavy quark self-energy and there is no relation to the effective coupling at all.

The effect of a residual mass term on the correlation function has an almost trivial structure. To see this, let us for a moment ignore all the complexities of the residual mass term and treat it as a number. Multiple insertions of $\delta m$ into a heavy quark line can be summarized by implementing $\delta m$ on the Lagrangian level as already done in Eq. (3.30), which modifies the heavy quark propagator to

$$
\frac{1+\not}{2} \frac{i}{v k-\delta m}
$$

Call $\Pi_{5}(\omega)$ the correlation function, computed from the Lagrangian (1.1) without a residual mass term and $\Pi_{5}^{\delta m}(\omega)$ the same object, computed from the Lagrangian in Eq. (3.30). Then

$$
\Pi_{5}^{\delta m}(\omega)=\Pi_{5}(\omega-\delta m),
$$

i.e. the sole effect of a residual mass of the effective heavy quark is to produce a shift of the momentum scale in the correlation function. The validity of Eq. (4.10) is obvious on physical grounds. Recall that $\omega$ is "measured" from the point $m_{Q}$ (if $p$ is the physical momentum of the meson, $v p=m_{Q}+\omega$ ). Thus different choices of $m_{Q}$, leading to different values of $\delta m$, simply shift the "zero point" of the momentum scale. If the predictions of HQET are to be invariant under the choice of $m_{Q}$, this can only result in a change of the argument of the correlation function. Nevertheless a diagrammatic proof of Eq. (4.10) might be useful. Let $q$ denote the residual momentum of the heavy 
quark, let $\omega=v q$ and consider an arbitrary diagram $\Gamma$ that contributes to the perturbative expansion of $\Pi_{5}^{\delta m}(\omega)$. Since all diagrams with heavy quark loops vanish identically, the only way the heavy quark can appear in $\Gamma$ is as a line that joins the two current insertions and emitting an arbitrary number $m-1$ of gluons. Now label the independent loop momenta of $\Gamma$ such that the heavy quark propagators carry momentum $k_{i}+q$, $i=1, \ldots, m$ and call $p_{j}$ the remaining loop momenta. With this assignment all other propagators are independent of $q$ and the diagram can be represented as

$$
\Gamma=\int \prod_{i=1}^{m} \mathrm{~d} k_{i} \prod_{j} \mathrm{~d} p_{j}\left(\prod_{i=1}^{m} \frac{1}{v k_{i}+\omega-\delta m}\right) \times \tilde{\Gamma}\left(k_{i}, p_{j}\right),
$$

where the remaining part $\tilde{\Gamma}$ of the diagram is independent of $\omega$ and $\delta m$. Therefore any diagram depends only on the combination $\omega-\delta m$, proving Eq. (4.10).

Formally, the residual mass term is an ambiguous quantity and the terms proportional to $(\delta m / \omega)^{n} \sim\left(\Lambda_{\mathrm{QCD}} / \omega\right)^{n}$ are present in Eq. (4.3) to render $\Pi_{5}^{\delta m}(\omega)$ unambiguous and well defined. In practice, summation of perturbative expansions is never performed, since only a few low-order terms of the series are available. Eq. (4.10) tells us that neglecting the UV renormalon divergences in the perturbative series can be equally interpreted as an uncertainty of order $\Lambda_{\mathrm{QCD}}$ in the momentum scale $\omega$ of the correlation function. In this sense, we say that the UV renormalons can be "summed up" to produce an ambiguity of scale in the HQET. Indeed, this scale ambiguity captures most concisely the physics reflected in these UV renormalons. Finally, their appearance can be traced back to the attempt to split a nonperturbative residual momentum $k$ of order $\Lambda_{\mathrm{QCD}}$ from the meson momentum, attributing the remainder to the momentum of a "physical" quark. This is not an infrared safe procedure, as is clearly visible from the IR renormalons in the pole mass.

At this point, comparison with the lattice formulation of HQET may help to clarify the meaning of an ambiguous residual mass. The discretized version of the heavy quark propagator has a linear divergence in the lattice spacing starting from first order in perturbation theory [20], which comes from the presence of a dimensionful cutoff and can be absorbed into a mass renormalization. The asymptotic behaviour of correlation functions at large times is proportional to $\exp (-\bar{\lambda} t)$, where $\bar{\lambda}$ is the mass of the lowest excitation of the theory (see Eq. (3.31)). The presence of a linear divergence leads to the conclusion [20] that the exponent $\vec{\lambda}$ is not a physical quantity. It is evident that the UV renormalons, the ambiguous residual mass term (and consequently $\bar{\lambda}$, see Sect. 3.3) and the scale ambiguity of correlation functions are in fact a reflection in the continuum of one and the same phenomenon that has been observed a long time ago on the lattice.

\section{The status of $Q C D$ sum rules}

The SDE of correlation functions has become an important tool to determine the various nonperturbative parameters of HQET through the QCD sum rules method. In the light of our results some of the common lore about the QCD sum rules, when applied to HQET, needs to be revised. It is instructive to trace uncertainties induced by 
the presence of UV renormalons in the SDE of correlation functions in HQET on some particular sum rules. We start with the simplest one, which is for the $B$-meson coupling in the static limit. An important result obtained from this sum rule is an estimate of the quantity $\bar{\Lambda}$ (or $\bar{\lambda}$ ), a task which has never been accomplished on the lattice for the reasons mentioned above.

In the QCD sum rule approach one takes the Borel transform of the SDE of the correlation function with respect to $1 / \omega$ (not to be confused with the Borel transform with respect to the coupling, which has been discussed in the previous sections), trading the frequency $\omega$ for a new variable, the Borel parameter $\tau$. This "theoretical" expression is matched to the "phenomenological" part of the sum rule, which uses a dispersive representation of the correlation function and saturation of the imaginary part by hadronic states. The Borel transformation serves several purposes, and ensures that both higher condensate contributions to the SDE and higher-mass contributions to the expansion of the imaginary part in hadron states are suppressed. The matching is performed in a certain intermediate region of the Borel parameter, where one hopes that both the SDE and the hadron expansion work reasonably well. The effect of the UV renormalons on the sum rule for the $B$-meson coupling (see, e.g., Refs. $[49,50]$ ) can easily be seen by the use of Eq. (4.10). Indeed, since by virtue of the Borel transformation

$$
\frac{1}{(\delta m-\omega)^{n}} \rightarrow \mathrm{e}^{-\delta m / \tau} \frac{1}{(n-1) ! \tau^{n}},
$$

the effect of the ambiguity in the scale $\omega$ transforms to an overall factor $\exp (-\delta m / \tau)$ in front of the "theoretical" side of the sum rule. Thus, to be concrete, the sum rule for the correlation function $\Pi_{5}$ in Eq. (4.1) is modified to

$$
\begin{aligned}
\widehat{f}_{B}^{2} \mathrm{e}^{-\bar{\Lambda} / \tau}= & \mathrm{e}^{-\delta m / \tau}\left\{\frac{3}{\pi^{2}} \int_{0}^{\omega_{0}} \mathrm{~d} \omega \omega^{2} \mathrm{e}^{-\omega / \tau}[1+\text { perturbative series }]\right. \\
& \left.-\langle\bar{q} q\rangle(\mu=2 \tau)+\frac{1}{16 \tau^{2}}\langle g \bar{q} \sigma G q\rangle(\mu=2 \tau)+\ldots\right\},
\end{aligned}
$$

where $\widehat{f}_{B}$ is the $B$-meson leptonic decay constant in the static limit (at a low scale $\mu=2 \tau)$, and $\omega_{0}$ is the duality interval for the lowest bound state. For simplicity, we have discarded the radiative corrections, see Ref. [49] for the complete expression to two-loop accuracy.

The factor $\exp (-\delta m / \tau)$ can be brought to the 1.h.s. and combined with $\exp (-\bar{\Lambda} / \tau)$ so that the sum rule depends on the combination $\bar{\Lambda}-\delta m$ only, as expected. Since this parameter is extracted from the sum rule by a fitting procedure, one may conjecture that the effects of renormalons are completely eliminated. As stressed repeatedly above, this conclusion is wrong. The presence of UV renormalons in the perturbative series on the r.h.s. of Eq. (5.2) indicates a principal ambiguity in its summation, which is expressed in a shorthand form by the appearance of the ambiguous residual mass term. In other words, if one calculated the corrections to the r.h.s. of the sum rule, Eq. (5.2), from large orders in the perturbative expansion (using some prescription to sum the series, see above, which also fixes $\delta m$ to a definite value), the main effect of these corrections 
will be a change of the output value of $\bar{\Lambda}-\delta m$ by an amount of order $A_{\mathrm{QCD}}$. Note that the coupling $\widehat{f}_{B}$ formally is protected from such corrections-the residue of the pole in the correlation function does not depend (formally) on the position of this pole. In practice, however, the values of $\bar{\Lambda}-\delta m$ and $\widehat{f}_{B}$ extracted from the sum rule are strongly correlated (see e.g. the discussion in Ref. [49]), and an uncertainty of $100 \mathrm{MeV}$ in $\bar{\Lambda}-\delta m$ induces an uncertainty of order $15 \%$ for the static decay constant.

The observation that the effect of the non-Borel-summable UV renormalons in HQET can generally be ascribed to an ambiguous residual mass term, allows for a back-on-theenvelope estimate of their importance in other sum rules, which have a more complicated structure. As an example, let us consider the sum rule for the heavy quark kinetic energy, which is defined by the expectation value of the operator of the nonrelativistic kinetic energy in the meson state. In the presence of a residual mass term it is given by

$$
K_{\delta m}=-\left\langle M(v)\left|\bar{h}_{v}(i D-\delta m)^{2} h_{v}\right| M(v)\right\rangle,
$$

where the nonrelativistic normalization of states $\langle M(v) \mid M(v)\rangle=1$ is implied ${ }^{21}$. As $\bar{\lambda}$, this matrix element is in fact independent of $\delta \mathrm{m}$. To derive the sum rule, one considers the correlation function

$$
\begin{gathered}
i^{2} \int \mathrm{d} x \int \mathrm{d} y \mathrm{e}^{i \omega(v \cdot x)-i \omega^{\prime}(v \cdot y)}\left\langle 0\left|j_{5}^{\dagger}(x) \vec{h}_{v}(0)(i D-\delta m)^{2} h_{v}(0) j_{5}(y)\right| 0\right\rangle \\
=T_{K}\left(\omega, \omega^{\prime}\right) .
\end{gathered}
$$

Assuming, as before, that the effect of ignoring the UV renormalon divergence in large orders is equivalent to an ambiguity in the external momenta (frequencies) and repeating a set of standard steps we arrive at the sum rule

$$
\begin{array}{r}
\widehat{f}_{B}^{2} K_{\delta m} \mathrm{e}^{-(\bar{A}-\delta m) / \tau}=\frac{3}{\pi^{2}} \int_{0}^{\omega_{0}} \mathrm{~d} \omega \omega^{4} \mathrm{e}^{-\omega / \tau}[1+\text { perturbative series }] \\
+\frac{1}{4} \tau\left(1-\mathrm{e}^{-\omega_{0} / \tau}\right)\left\langle\frac{\alpha}{\pi} G G\right\rangle-\frac{3}{8}\langle g \bar{q} \sigma G q\rangle(\mu=2 \tau)+\ldots
\end{array}
$$

Again, for simplicity we have discarded the radiative corrections calculated in Ref. [30].

In Eq. (5.5) we recognize the familiar source of ambiguity related to an uncertainty in the position $\bar{A}-\delta m$ of the ground state. However, an additional uncertainty is present already in the definition of the matrix element in Eq. (5.3) due to quadratic and linear UV divergences, cf. Ref. [20]. With respect to quadratic divergences it is interesting to note that the corresponding IR renormalon in the pole mass at $u=1$ is absent, see Eq. (3.13). In any case, the sum rule analysis in Ref. [30] has yielded a relatively large value for $K$, of order $0.6 \mathrm{GeV}^{2}$, which may indicate that the kinetic energy has a large "genuinely nonperturbative" contribution, not related to renormalons, and in this respect is similar to the gluon condensate.

\footnotetext{
${ }^{21}$ We have changed the sign in the definition compared to Ref. [30]. In the conventional notation [29] $K=-\lambda_{1}$.
} 
To summarize, the QCD sum rule approach faces precisely the same difficulties in defining the observables of HQET, which have been recognized in studies of HQET on the lattice. However, there is also a difference. In lattice calculations one does not distinguish between perturbative and nonperturbative contributions to the correlation functions. Thus the renormalon problem is difficult to overcome, see Ref. [20]. In QCD sum rules one isolates the "genuinely nonperturbative" contributions in a few parameters, the vacuum condensates, which are determined from phenomenology. In spite of the fact that such an approach can not be fully consistent theoretically-the condensates can never be determined to arbitrary accuracy without running into the renormalon problem or without the introduction of a "hard" factorization scale-it may nevertheless be quite successful phenomenologically, as it has been in the application to light quarks. A novel feature of the QCD sum rules in HQET is that the ambiguity in the separation of the perturbative and nonperturbative contributions affects not only the values of condensates on the "theoretical" side of the sum rule, but also the quantities that enter the "phenomenological" side. In the HQET, the 1.h.s. of sum rules like Eq. (5.5) is only fully defined after one has dealt with the UV renormalons in the perturbative expansions on the r.h.s., though in practice one might hope to be as lucky as in QCD, where the renormalons can be ignored, since the "true" nonperturbative contributions to theoretically ambiguous quantities turn out to be large. Thus, for the practitioner, the appearance of an ambiguous residual mass of order (100-200) $\mathrm{MeV}$, see Eq. (3.17), can serve as an error bar on the determination of quantities like $\bar{\Lambda}$.

\section{Conclusions}

The investigation of the asymptotic behaviour of perturbative expansions in HQET reveals that in addition to the IR renormalon divergence, which can be related to condensates in the SDE, the correlation functions possess non-summable UV renormalons. These UV renormalons are not related to a Landau ghost as familiar from QED, but rather indicate a fine-tuning problem of HQET. The natural mass of the effective heavy quark is $m_{Q}$ (and not zero), a fact that is obscured by the use of dimensional regularization, which does not introduce a dimensionful parameter, as long as poles at $d=4$ only are subtracted. The UV renormalons reflect a linear divergence of the self-energy of the heavy quark, which is seen already in perturbation theory, when a dimensionful cutoff is employed. The absence of a dimensionful quantity in the leading effective Lagrangian of HQET is fake and if one attempts to go beyond perturbation theory, the residual mass arises necessarily as a second parameter in the Lagrangian. This is more evident, when one does not consider HQET as a quantum field theory in its own right, but embedded in QCD, whose heavy mass limit it is supposed to extract. To avoid the UV renormalon problem, one can not use the standard dimensional renormalization, which yields only an incomplete factorization of effects on different distance scales on the level of logarithms. Technically, complete factorization can be achieved by a "hard" cutoff, which is very awkward for practical calculations. As an alternative, we have indicated a factorization procedure for renormalons, which on the level of the HQET Lagrangian corresponds to a mass term proportional to the scale $\mu$. 
The fine-tuning problem of HQET has a very transparent interpretation, when it is viewed from the perspective of full QCD. HQET (to leading order in $m_{Q}$ ) is a theory for light quarks in the field of a static colour source. In perturbation theory, this notion does not present a difficulty. One may imagine the light quark removed to an infinite distance from the source and include the energy of the field of the heavy quark into a renormalization of its mass. In this way, the pole mass emerges naturally as the parameter to be used in the heavy quark expansion. Beyond perturbation theory, this operational definition looses its meaning due to confinement. The meson is an indivisible entity (for QCD) and an unambiguous separation of an energy of the field and a binding energy of the light quark in this field can not be performed. Remarkably, perturbation theory knows about this problem and reveals it as an IR renormalon in the pole mass. The position of this renormalon in the Borel plane fixes the inherent ambiguity in the concept of a pole mass to be of order $A_{\mathrm{QCD}}$. From their physical origin, it is clear that these IR renormalons are very different from the ones encountered in the SDE and, in particular, they do not correspond to any condensate.

Nonetheless, the implications of these IR renormalons for the structure of the heavy mass expansion are very close conceptually to their namesakes in the SDE. First, the UV renormalons in the correlation functions of HQET reflect in fact one and the same phenomenon as the IR renormalon in the pole mass. If we assume that the Green functions of QCD can be reconstructed from an extended (and presumably very intricate) Borel summation procedure-a conjecture, of course! - then it is very natural to remedy the ambiguities of correlation functions in HQET from the UV singularities by the inclusion of an ambiguous residual mass into the Lagrangian. This leads immediately to the conclusion that parameters like $\bar{\Lambda}$ (or $\bar{\lambda}$, to be precise) are not physical quantities, but in fact ambiguous. This is indeed a necessity, because these parameters arise in power-suppressed (in $1 / m_{Q}$ ) terms of the heavy mass expansion, whose leading term has a divergent perturbative expansion. In this respect the heavy mass expansion is in complete analogy with the SDE, where the role of $\bar{\lambda}$ etc. is played by the condensates, whose theoretically ambiguous status has been realized a long time ago. In this light the appearance of an IR renormalon in the pole mass at $t=-1 /\left(2 \beta_{0}\right)$, which is closer to the origin of the Borel plane and implies a stronger divergence of perturbative series than in the SDE, is very natural, since $1 / m_{Q}$ corrections are present in the heavy quark expansion and are parametrized by $\bar{\lambda}$.

Since the interpretation of the various quantities that appear in asymptotic expansions with exponentially small (in the coupling) components such as the SDE or the heavy quark expansion has caused confusions in the past (see the discussion of this point in Refs. [15,38]), which are merely a problem of language, it might be useful to recall that there are two attitudes concerning the renormalon problem, which already have been alluded to in Sect. 2: First, one can interpret these expansions as asymptotic expansions in the mathematical sense ${ }^{22}$. Then one faces the problem of divergent series, their summation and the Stokes discontinuities in the exponentially small components, which leads to the notion of formally ambiguous parameters. Second, one might follow Wilson's operator product expansion literally and introduce a hard factorization scale

$\overline{22}$ Assuming, of course, that they are asymptotic to something. 
$\mu$. In this way, the divergence of perturbative expansions is eliminated at the price of parameters that depend explicitly on the scale $\mu$. Both approaches are of course equivalent in their physical content: The quantities in the power-suppressed terms are not physical in the sense that they can not be determined to arbitrary accuracy without further specification. In the first approach this is a prescription to sum the divergent series in the leading terms (a principal value prescription, for instance), in the second, quantities like $\bar{\lambda}$ in the heavy quark expansion and the gluon condensate in the SDE depend power-like on the factorization scale. Both approaches are also impractical: Neither can we sum perturbative expansions in view of the few low-order terms that are generally available, nor can we calculate Feynman diagrams with an explicit cutoff. Thus, although the second approach looks much more natural to phenomenology, where one is prepared to fit the unknown quantities anyway, one has to rely in both cases on the hope that "true" nonperturbative contributions turn out to be large. If nature likes it different, the study of power corrections is academic anyway and one should devote oneself to the calculation of the next unknown order of perturbation theory.

We have chosen the first approach in the present paper because of the universality of the UV renormalons in HQET. They arise only through the linear divergence of the heavy quark self-energy. After we include the formally ambiguous mass, we can easily trace the effects of the UV renormalons through the appearance of the residual mass term in the matrix elements and operators of HQET.

The universality of the phenomenon is also important to recognize its phenomenological consequences. The inclusive $B$-decay widths are a prime example of practical interest. The ambiguity of order $\Lambda_{\mathrm{QCD}}$ in the pole mass implies that when parametrized in terms of the pole mass, the theoretical prediction for the absolute widths can not be better than terms of order $\Lambda_{\mathrm{QCD}} / m_{b}$. However, the IR renormalon in the leading term is universal for all $B$-hadrons and cancels in the difference of the widths, which indeed scale with the heavy quark mass as $\Lambda_{\mathrm{QCD}}^{2} / \mathrm{m}_{b}^{2}$.

As a second example, the status of the pole mass itself warrants discussion. For phenomenology, the most important question is how large the intrinsic uncertainty of the pole mass could be numerically. Our estimate from the divergence of the perturbative expansion suggests values in the range $\delta m_{\text {pole }} \sim 170-280 \mathrm{MeV}$, but this can only be an order-of-magnitude guess. There are various indications from phenomenology that the actual ambiguity is indeed of this order or rather smaller. All existing phenomenological analyses of the $b$-quark pole mass fall in the range $4.55-4.85 \mathrm{GeV}$, a fraction of which can well be ascribed to an inherent ambiguity of the concept "pole mass". In this context, it is interesting to note that the existing calculations of the quantity $\bar{A}$ in HQET give $\bar{\Lambda}=400-600 \mathrm{MeV}[49,50]$ with an uncertainty of the same order as for the pole mass. Last but not least, it has been pointed out [51], that a meson with a light and an infinitely heavy quark might provide a definition of the constituent quark-one of the most mysterious objects in QCD. Indeed, the correlation function of two heavy-light currents, Eq. (4.1), may be rewritten as the vacuum expectation value of the nonlocal operator [52] 


$$
\left\langle 0\left|\bar{q}(x) \mathrm{P} \exp \left[i g \int_{0}^{1} \mathrm{~d} u x_{\mu} A_{\mu}(u x)\right] q(0)\right| 0\right\rangle \stackrel{x^{2} \rightarrow-\infty}{\sim} \exp \left[-\bar{\Lambda} \sqrt{-x^{2}}\right],
$$

which gives a natural definition of the propagator of a constituent quark, so that $\bar{A}$ may be interpreted as the constituent quark mass. The celebrated successes of nonrelativistic quark models (for light quarks) have not found any rational explanation so far, but indicate rather strongly that the mass of the constituent quark is phenomenologically stable and of order $350 \mathrm{MeV}$. This falls into the range of values quoted for $\bar{A}$ to $100-200 \mathrm{MeV}$ accuracy. Combining these estimates from different branches of phenomenology, we should conclude that there is a lot of indirect evidence, that the difference between the hadron mass and the quark pole mass in the heavy quark limit has a large "genuinely" nonperturbative contribution and the uncertainty of the concept of the pole mass is likely to stay within $100-200 \mathrm{MeV}$.

\section{Acknowledgement}

It is a pleasure to thank V.I. Zakharov for many interesting discussions related to the subject of this paper. M.B. wishes to thank M. Einhorn for an instructive conversation. V.B. gratefully acknowledges discussions with N.G. Uraltsev, which initiated this study, and our special thanks are to him for sending us a preliminary version of Ref. [47]. We acknowledge an overlap with some of the results and conclusions of this paper.

\section{Appendix A. Renormalization of the Borel transform}

In this appendix we prove that renormalization of the divergence associated with the integration over the gluon momentum amounts to subtracting the pole term of the Borel transform at $u=0$ plus some finite terms which depend on the renormalization scheme. As a by-product, we find the anomalous dimension of the heavy quark field to leading order in $1 / N_{f}$. The derivation is given for the simplest case of the heavy quark self-energy, but proceeds almost identically for a massive quark or the vertex function.

We start $a b$ initio and compute the regularized coefficient of the heavy quark selfenergy in order $a^{n+1}$. We use dimensional regularization in $d=4+2 \epsilon$ dimensions. A straightforward calculation of the diagram of Fig. 6a yields

$$
p_{n}^{\mathrm{reg}}(d)=\frac{C_{\mathrm{F}}}{4 \pi N_{f}} v k \beta_{0}^{n} \frac{1}{(n+1) \epsilon^{n+1}} G(d,(n+1) \epsilon),
$$

where $\beta_{0}=1 /(6 \pi), C_{\mathrm{F}}=4 / 3$ and $G(d, s)$ is given by

$$
\begin{aligned}
G(d, s)= & \left(\frac{1}{4 \pi}\right)^{s}\left(-\frac{2 v k}{\mu}\right)^{2 s}\left[-6 \epsilon \frac{\Gamma(-\epsilon) \Gamma(2+\epsilon)^{2}}{\Gamma(4+2 \epsilon)}\right]^{s / \epsilon-1} \\
& \times(-2 s)(3+2 \epsilon) \frac{\Gamma(-1-2 s) \Gamma(1+s)}{\Gamma(2+\epsilon-s)}
\end{aligned}
$$




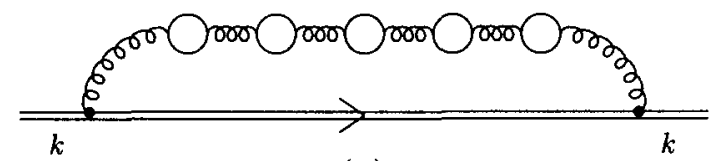

(a)

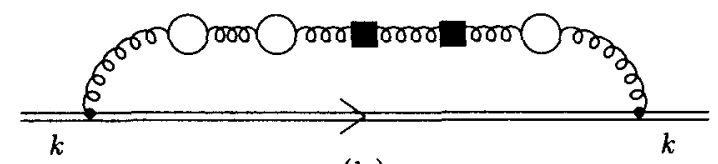

(b)

Fig. A.1. (a) Diagram with $n$ fermion loops contributing to the self-energy to order $a^{n+1}$. (b) Counter-diagram with $k$ bubbles replaced by counterterms.

For later use we collect the definitions

$$
G(d, s)=\sum_{j=0}^{\infty} G_{j}(d) s^{j}, \quad G_{0}(d)=\sum_{j=0}^{\infty} g_{j} \epsilon^{j}
$$

with

$$
\begin{aligned}
& G(d, 0)=G_{0}(d)=-\frac{1}{6}(3+2 \epsilon) \frac{\Gamma(4+2 \epsilon)}{\Gamma(1-\epsilon) \Gamma(2+\epsilon)^{3}}=-3-4 \epsilon+O\left(\epsilon^{2}\right), \\
& G(4, s)=\left(-\frac{2 v k}{\mu}\right)^{2 s} \mathrm{e}^{s\left(\gamma_{\mathrm{E}}-5 / 3-\ln 4 \pi\right)}(-6 s) \frac{\Gamma(-1-2 s) \Gamma(1+s)}{\Gamma(2-s)} .
\end{aligned}
$$

$\gamma_{\mathrm{E}}$ denotes the Euler-Mascheroni constant. The limits $d \rightarrow 4$ and $s \rightarrow 0$ do not commute in the general case (although they do for the heavy quark self-energy considered here).

The diagram in Fig. 6a consists of two basic renormalization parts: The fermion bubble and the diagram itself. The counterterm for a fermion bubble is given by $-\beta_{0}(1 / \epsilon+$ finite $)$. We use first the minimal subtraction scheme and comment on other renormalization schemes later. Thus we do not subtract finite terms. Take now the diagram in Fig. 6b, where $k$ bubbles have been replaced by their counterterms. Since the only dependence on $n$ on the r.h.s. of Eq. (A.1) originates from the number of fermion loops, substitute $n \rightarrow n-k$ and multiply by $\left(-\beta_{0} / \epsilon\right)^{k}$ for each of the $k$ counterterms. Finally account for a combinatorical factor $n ! /(k !(n-k) !)$ according to the number of ways, $k$ bubbles can be picked from the $n$ bubbles available. As a result of these manipulations a partially renormalized coefficient, incorporating the renormalization of the coupling $a$, is obtained:

$$
\begin{aligned}
p_{n}^{\text {part.ren }}(d) & =\frac{C_{\mathrm{F}}}{4 \pi N_{f}} v k \beta_{0}^{n} \sum_{k=0}^{n} \frac{1}{\epsilon^{n+1}} \frac{(-1)^{k}}{n+1-k}\left(\begin{array}{l}
n \\
k
\end{array}\right) G(d,(n+1-k) \epsilon) \\
& =\frac{C_{\mathrm{F}}}{4 \pi N_{f}} v k \beta_{0}^{n} \sum_{j=0}^{n+1} \frac{G_{j}(d)}{\epsilon^{n+1-j}} \sum_{k=0}^{n}(-1)^{k}\left(\begin{array}{l}
n \\
k
\end{array}\right)(n+1-k)^{j-1}+O(\epsilon) .
\end{aligned}
$$


The sum over $j$ is truncated at $n+1$, because we are not interested in terms that vanish, when $\epsilon$ is taken to zero. The sum over $k$ can be taken. It is non-zero only for $j=0$ and $j=n+1$, a simplification that was first observed in Ref. [53]. Thus

$$
p_{n}^{\text {part.ren }}(d)=\frac{C_{\mathrm{F}}}{4 \pi N_{f}} v k \beta_{0}^{n}\left[\frac{(-1)^{n}}{n+1} \frac{1}{\epsilon^{n+1}} G_{0}(d)+n ! G_{n+1}(4)+O(\epsilon)\right] .
$$

In the next step we account for the subtraction for the whole diagram and then take $\epsilon=0$. It is gratifying that only the finite terms of $p_{n}^{\text {part.ren }}(d)$ depend on $v k / \mu$, as it must be. The renormalized coefficient in the MS scheme is then given by

$$
p_{n}=\frac{C_{\mathrm{F}}}{4 \pi N_{f}} v k \beta_{0}^{n}\left[n ! G_{n+1}(4)+\frac{(-1)^{n}}{n+1} g_{n+1}\right] \text {. }
$$

It is easy to see that the $g_{n}$ do not diverge factorially, because $G_{0}(d)$ is analytic at $d=4$. Finally, we go over to the Borel transform of the renormalized self-energy and find

$$
\begin{aligned}
& \tilde{\Sigma}_{\mathrm{eff}}(v k, u)=\sum_{n=0}^{\infty} p_{n} \frac{t^{n}}{n !}=\frac{\epsilon_{\mathrm{F}}}{4 \pi N_{f}} v k\left[\frac{G(4,-u)-G_{0}(4)}{(-u)}+R(u)\right] \\
& =\frac{C_{\mathrm{F}}}{4 \pi N_{f}} v k\left[\left(-\frac{2 v k}{\mu}\right)^{-2 u} \mathrm{e}^{-u\left(\gamma_{\mathrm{E}}-5 / 3-\ln 4 \pi\right)}(-6) \frac{\Gamma(-1+2 u) \Gamma(1-u)}{\Gamma(2+u)}-\frac{3}{u}+R(u)\right],
\end{aligned}
$$

where

$$
R(u)=\sum_{n=0}^{\infty} \frac{1}{(n+1) !} g_{n+1} u^{n}=\frac{3}{u}+\frac{\tilde{G}_{0}(u)}{u}
$$

is an entire function. $\tilde{G}_{0}$ denotes the Borel transform of $G_{0}$ given in Eq. (A.4). As promised, the renormalized self-energy differs from the partially renormalized one, obtained from insertion of the Borel-transformed gluon propagator, Eq. (2.11), only by subtraction of the pole at $u=0$ and scheme-dependent finite terms.

Let us dwell more on the issue of scheme dependence. Eq. (A.8) is easily generalized to arbitrary minimal subtraction-like schemes such as $\overline{\mathrm{MS}}$. These schemes have identical renormalization group functions and differ only by a global scale change. To the order of the $1 / N_{f}$ expansion considered here, the difference resides in the finite term $C$ of the fermion bubble. Observing that in the MS scheme $C=\gamma_{E}-5 / 3-\ln 4 \pi$, we obtain Eq. (A.8) in an arbitrary MS-like scheme by the replacement

$$
\mathrm{e}^{-u\left(\gamma_{E}-5 / 3-\ln 4 \pi\right)} \longrightarrow \mathrm{e}^{-u C}
$$

The function $R(u)$ is the same for all MS-like schemes. In principle, the finite renormalizations and therefore $R(u)$ can be chosen arbitrarily. In momentum subtraction schemes the finite terms are factorially divergent and $R(u)$ is no longer an entire function. This 
introduces a divergence into the perturbative expansion of the renormalization group functions, which hides part of the divergence of the perturbative series in the definition of the renormalized parameters of the theory. For this reason the choice of such schemes is disfavoured, when one considers large orders in perturbation theory [36]. We will always assume that a scheme is chosen, where the counterterms are analytic in $\alpha$ near $\alpha=0$, in which case the function $R(u)$ is entire in the Borel plane and the renormalized parameters are unambiguously defined in terms of the bare parameters. In the general situation of the self-energy of a massive quark $R$ can also depend on the mass. We restrict ourselves to mass-independent schemes.

We conclude this appendix with the anomalous dimension of the heavy quark field in MS-like schemes. The bare field is related to the renormalized one through $h_{v}^{0}=Z^{1 / 2} h_{v}$. From Eq. (A.6) we get

$$
Z^{-1}=1+\frac{a(\mu)}{4 \pi N_{f}} C_{\mathrm{F}} \sum_{n=0}^{\infty}\left(\beta_{0} a(\mu)\right)^{n} \frac{(-1)^{n}}{(n+1) \epsilon^{n+1}} G_{0}(d)+\text { finite }+O\left(\frac{1}{N_{f}^{2}}\right)
$$

The anomalous dimension of the field is defined by

$$
\gamma(a) \equiv \mu^{2} \frac{\partial Z}{\partial \mu^{2}}=\lim _{\epsilon \rightarrow 0} \beta(\epsilon, a) \frac{\partial Z}{\partial a} .
$$

Recalling $\beta(\epsilon, a)=\epsilon a+\beta_{0} a^{2}+O\left(1 / N_{f}\right)$, a short calculation yields

$$
\gamma(a)=\frac{a}{4 \pi N_{f}} C_{\mathrm{F}} G_{0}\left(4-\beta_{0} a\right)+O\left(\frac{1}{N_{f}^{2}}\right) .
$$

We remind the reader that the anomalous dimension is gauge dependent and the result is given in the Landau gauge. However, one may easily check that to order $1 / N_{f}$ only the $O(a)$-coefficient is gauge dependent. As expected in MS-like schemes, the anomalous dimension has a finite radius of convergence in $a$. Comparison with Eq. (A.9) shows that the finite renormalizations in a given scheme are essentially the Borel transform of the anomalous dimension.

\section{Appendix B. The scalar two-loop integral}

The aim of this appendix is to find an expression for the scalar integral

$$
\begin{aligned}
& S(d, \gamma)=\frac{(4 \pi)^{d}}{4(2 \omega)^{-2 \gamma}} \int \frac{d^{d} k}{(2 \pi)^{d}} \frac{d^{d} p}{(2 \pi)^{d}}\left(-\frac{1}{k^{2}}\right)\left(-\frac{1}{p^{2}}\right) \\
& \times\left(-\frac{1}{(k-p)^{2}}\right)^{\gamma}\left(\frac{1}{v k+\omega}\right)\left(\frac{1}{v p+\omega}\right)
\end{aligned}
$$

with arbitrary complex $\gamma$, which is suited to extract its analyticity properties. The prefactor is chosen for later convenience. The first step is to rewrite this integral in 
coordinate space and to apply the Gegenbauer polynomial technique [54]. Following the by now standard procedure, we arrive at

$$
\begin{aligned}
S(d, \gamma)= & 2 \frac{\Gamma(d / 2-1) \Gamma(d / 2-\gamma-1) \Gamma(6+2 \gamma-2 d)}{\Gamma(d-2)} P(d, \gamma), \\
P(d, \gamma)= & \frac{1}{\Gamma(\gamma)} \sum_{n=0}^{\infty} \frac{(-1)^{n}}{n !} \Gamma(d-2+n) \\
& \times \int_{0}^{1 / 2} \mathrm{~d} u\left(\frac{1}{n+\gamma} u^{2+2 \gamma-d+n} \bar{u}^{2-d-n}+\frac{1}{\gamma-d-n+2} u^{n} \bar{u}^{2 \gamma-2 d+4-n}\right)
\end{aligned}
$$

with $\bar{u} \equiv 1-u$. The summation can be taken at the price of another integration and yields

$$
P(d, \gamma)=\frac{\Gamma(d-2)}{\Gamma(\gamma)} \int_{0}^{1 / 2} \mathrm{~d} u \int_{0}^{1} \mathrm{~d} t \frac{t^{d-3-\gamma}}{(1-u \bar{t})^{d-2}}\left\{(t u)^{2-d+2 \gamma}-\bar{u}^{2-d+2 \gamma}\right\} .
$$

Next, the denominator is expanded, which allows us to perform the $t$-integration:

$$
\begin{aligned}
P(d, \gamma)= & \sum_{n=0}^{\infty} \frac{1}{3-d+2 \gamma+n}\left(\frac{1}{2}\right)^{3-d+2 \gamma+n} \frac{\Gamma(d-2+n)}{\Gamma(n+\gamma+1)} \\
& -\frac{\Gamma(d-2)}{(d-2-\gamma) \Gamma(\gamma)} \int_{0}^{1 / 2} \mathrm{~d} u \bar{u}^{2-d+2 \gamma}{ }_{2} F_{1}(d-2,1, d-1-\gamma ; u),
\end{aligned}
$$

where ${ }_{2} F_{1}$ is the hypergeometric function. Now we do some juggling with the hypergeometric function in order to replace the hypergeometric function in this equation by hypergeometric functions with the argument $\bar{u}$. When this is done, part of the $u$-integrals can be done. Further, we note that ${ }_{2} F_{1}(1-\gamma, d-2-\gamma, 1-\gamma ; \bar{u})=u^{2-d+\gamma}$ and obtain

$$
P(d, \gamma)=\sum_{n=0}^{\infty} \frac{1}{3-d+2 \gamma+n} \frac{\Gamma(d-2+n)}{\Gamma(n+\gamma+1)}-\Gamma(d-2-\gamma) \int_{0}^{1 / 2} \mathrm{~d} u(u \bar{u})^{2-d+\gamma} .
$$

The integral is elementary and the sum can be expressed in terms of the generalized hypergeometric function ${ }_{3} F_{2}$. Thus

$$
\begin{aligned}
P(d, \gamma)= & -\frac{1}{2} \frac{\Gamma(1-s) \Gamma(s)^{2}}{\Gamma(2 s)} \\
& +\frac{\Gamma(\gamma+1-s)}{(s+\gamma) \Gamma(\gamma+1)}{ }_{3} F_{2}(\gamma+1-s, 1, s+\gamma ; \gamma+1, \gamma+1+s ; 1),
\end{aligned}
$$


where $s \equiv 3-d+\gamma$ and the series definition of the ${ }_{3} F_{2}$ function is

$$
\begin{aligned}
& { }_{3} F_{2}(a, b, c ; \alpha, \beta ; z) \\
& \quad=\sum_{n=0}^{\infty} \frac{\Gamma(a+n)}{\Gamma(a)} \frac{\Gamma(b+n)}{\Gamma(b)} \frac{\Gamma(c+n)}{\Gamma(c)} \frac{\Gamma(\alpha)}{\Gamma(\alpha+n)} \frac{\Gamma(\beta)}{\Gamma(\beta+n)} \frac{z^{n}}{n !} .
\end{aligned}
$$

The series, when applied to Eq. (B.7), converges for $y>d-3$ only, which is not yet sufficient for our purpose. We therefore use the identity [55]

$$
\begin{aligned}
& { }_{3} F_{2}(\gamma+1-s, 1, s+\gamma ; \gamma+1, \gamma+1+s ; 1) \\
& \quad=\frac{\Gamma(\gamma+1) \Gamma(\gamma+1+s)}{s \Gamma(\gamma+1-s) \Gamma(2 s+\gamma)}{ }_{3} F_{2}(s, 2 s, s ; s+1,2 s+\gamma ; 1),
\end{aligned}
$$

which provides a representation that converges for any $\gamma$, as long as $d>2$. The final answer for $S(d, \gamma)$ is

$$
\begin{aligned}
S(d, \gamma)= & \frac{\Gamma(d / 2-1) \Gamma(d / 2-\gamma-1)}{\Gamma(d-2)}\left\{-\Gamma(1-s) \Gamma(s)^{2}\right. \\
& \left.+2 \frac{\Gamma(s+\gamma) \Gamma(2 s)}{s \Gamma(2 s+\gamma)}{ }_{3} F_{2}(s, s, 2 s ; s+1,2 s+\gamma ; 1)\right\} .
\end{aligned}
$$

In four dimensions $S(4, \gamma)$ is meromorphic with poles at all integers, negative halfintegers and $\gamma=1 / 2$. Useful expansions about some of the singularities in four dimensions are

$$
\begin{array}{rlrl}
S(4,-1 / 2+\delta) & =-\frac{1}{3 \delta^{2}}+O\left(\frac{1}{\delta}\right), & S(4, \delta) & =\frac{1}{2 \delta^{2}}+\frac{1}{2 \delta}+O(1), \\
S(4,1 / 2+\delta) & =-\frac{1}{\delta^{2}}+O\left(\frac{1}{\delta}\right), & S(4,1+\delta) & =\frac{\pi^{2}}{3 \delta}+O(1), \\
S(4,2+\delta) & =\frac{1}{\delta^{2}}+O(1), & S(4,3+\delta) & =\frac{1}{2 \delta^{2}}-\frac{2}{3 \delta}+O(1), \\
S(4,4+\delta) & =\frac{1}{3 \delta^{2}}+\frac{3}{10 \delta}+O(1), &
\end{array}
$$

\section{References}

[1] E. Eichten and B. Hill, Phys. Lett. B 234 (1990) 511

[2] B. Grinstein, Nucl. Phys. B 339 (1990) 253

[3] H. Georgi, Phys. Lett. B 240 (1990) 447

[4] N. Isgur and M.B. Wise, Phys. Lett. B 232 (1989) 113; B 237 (1990) 527

[5] G.P. Korchemsky and A.V. Radyushkin, Phys. Lett. B 279 (1992) 359 
[6] A.M. Polyakov, Nucl. Phys. B 164 (1980) 171

[7] V.S. Dotsenko and S.N. Vergeles, Nucl. Phys. B 169 (1980) 527

[8] R.A. Brandt, F. Neri and M.-a. Sato, Phys. Rev. D 24 (1981) 879

[9] H. Dorn, Fortschr. Phys. 34 (1986) 11

| 10| J. Gervais and A. Neveu, Nucl. Phys. B 163 (1980) 189

[11] I.Ya. Aref'eva, Phys. Lett. B 93 (1980) 347

[12] E. Bagan and P. Gosdzinsky, Phys. Lett. B 320 (1994) 123

[13] G. 't Hooft, in The Whys of Subnuclear Physics, Proc. Int. School, Erice, 1977, ed. A. Zichichi (Plenum, New York, 1978)

114] B. Lautrup, Phys. Lett. B 69 (1977) 438

[15] F. David, Nucl. Phys. B 234 (1984) 237; B 263 (1986) 637

[16] A.F. Falk, M. Neubert and M. Luke, Nucl. Phys. B 388 (1992) 363

[17] I.I. Bigi and N.G. Uraltsev, Phys. Lett. B 321 (1994) 412

[18] A. DiGiacomo and G.C. Rossi, Phys. Lett. B 100 (1981) 481

[19] T. Banks et. al., Nucl. Phys. B 190 [FS3] (1981) 692

[20] L. Maiani, G. Martinelli and C.T. Sachrajda, Nucl. Phys. B 368 (1992) 281

[21] J. Chay, H. Georgi and B. Grinstein, Phys. Lett. B 247 (1990) 399

|22| I. Bigi, N. Uraltsev and A. Vainshtein, Phys. Lett. B 293 (1992) 430 [Erratum: B 297 (1993) 477]

[23| A.V. Manohar and M.B. Wise, Phys. Rev. D 49 (1994) 1310

[24] M. Luke and M.J. Savage, Phys. Lett. B 321 (1994) 88

[25] M.B. Voloshin, preprint ITEP-21, Moscow (1980);

M.B. Voloshin and Yu.M. Zaitsev, Sov. Phys. Usp. 30 (1987) 553

[26] L.J. Reinders, Phys. Rev. D 38 (1988) 947

[27] S. Narison, Phys. Lett. B 197 (1987) 405

[28] M.A. Shifman, A.I. Vainshtein and V.I. Zakharov, Nucl. Phys. B 147 (1979) 385; For a recent review see M.A. Shifman, ed., Vacuum structure and QCD sum rules, Current Physics Sources and Comments, Vol. 10 (North-Holland, Amsterdam, 1992)

[29] M. Neubert, SLAC report SLAC-PUB-6263, June 1993, to appear in Phys. Rep.

[30] P. Ball and V.M. Braun, Phys. Rev. D 49 (1994) 2472

[31] A.H. Mueller, in QCD-Twenty Years Later, Proc. Int. Conf., Aachen, 1992, ed. P. Zerwas and H. Kastrup, Vol. 1 (World Scientific, Singapore, 1993)

[32] V.I. Zakharov, Nucl. Phys. B 385 (1992) 452

I33] A.D. Sokal, J. Math. Phys. 21(2) (1980) 261

[34] G. Parisi, Nucl. Phys. B 150 (1979) 163

135] A.H. Mueller, Nucl. Phys. B 250 (1985) 327

[36] M. Beneke, Phys. Lett. B 307 (1993) 154

[37] See, e.g., R.B. Dingle, Asymptotic expansions: their derivation and interpretation (Academic Press, London, 1973)

[38] V.A. Novikov et al., Phys. Rep. 116 (1984) 104; Nucl. Phys. B 249 (1985) 445

[39] M. Beneke and V.I. Zakharov, Phys. Lett. B 312 (1993) 340

[40] M.A. Shifman, in QCD-Twenty Years Later, Proc. Int. Conf., Aachen, 1992, ed. P. Zerwas and H Kastrup, Vol. 1 (World Scientific, Singapore, 1993)

[41] F. David, J. Feldman and V. Rivasseau, Commun. Math. Phys. 116 (1988) 215

[42] M. Beneke, Nucl. Phys. B 405 (1993) 424; PhD Thesis, München, 1993

[43] D. Broadhurst, Z. Phys. C 58 (1993) 339

144] M. Aguilar-Benitez et al., Review of Particle Properties, Phys. Rev. D 45 (1992) Part 2

[45] W. Celmaster and R.J. Gonsalves, Phys. Rev. D 20 (1979) 1420

[46] M. Beneke and V.I. Zakharov, Phys. Rev. Lett. 69 (1992) 2472

[47] I. Bigi et al., preprint TPI-MINN-94/4-T

[48] H. Georgi, B. Grinstein and M.B. Wise, Phys. Lett. B 252 (1990) 456

[49] E. Bagan et al., Phys. Lett. B 278 (1992) 457 
[50] M. Neubert, Phys. Rev. D 45 (1992) 2451; D 46 (1992) 1076

[51] J.D. Bjorken, Acta Phys. Pol. B 23 (1992) 637

[52] A.V. Radyushkin, Phys. Lett. B 271 (1991) 218

[53] A. Palanques-Mestre and P. Pascual, Commun. Math. Phys. 95 (1984) 277

[54] K.G. Chetyrkin, A.L. Kataev and F.V. Tkachov, Nucl. Phys. B 174 (1980) 345

[55] A.P. Prudnikov, Yu.A. Brychkov and O.I. Marichev, Integrals and Series, Vol. 3 (Nauka, Moscow, 1986) 